\title{
APPROXIMATION OF BOUNDARY CONTROL PROBLEMS ON CURVED DOMAINS*
}

\author{
EDUARDO $\mathrm{CASAS}^{\dagger}$ AND JAN SOKOLOWSKI
}

\begin{abstract}
In this paper we consider boundary control problems associated to a semilinear elliptic equation defined in a curved domain $\Omega$. The Dirichlet and Neumann cases are analyzed. To deal with the numerical analysis of these problems, the approximation of $\Omega$ by an appropriate domain $\Omega_{h}$ (typically polygonal) is required. Here we do not consider the numerical approximation of the control problems. Instead, we formulate the corresponding infinite dimensional control problems in $\Omega_{h}$, and we study the influence of the replacement of $\Omega$ by $\Omega_{h}$ on the solutions of the control problems. Our goal is to compare the optimal controls defined on $\Gamma=\partial \Omega$ with those defined on $\Gamma_{h}=\partial \Omega_{h}$ and to derive some error estimates. The use of a convenient parametrization of the boundary is needed for such estimates.
\end{abstract}

Key words. Neumann control, Dirichlet control, curved domains, error estimates, semilinear elliptic equations, second order optimality conditions

AMS subject classifications. 49J20, 35J65

DOI. $10.1137 / 090761550$

1. Introduction. In this paper we study boundary control problems defined on a curved domain $\Omega$. We start with the Neumann problem (NP) and consider the Dirichlet problem (DP) afterward. To numerically solve these problems, usually it is convenient to approximate $\Omega$ by a polygonal domain $\Omega_{h}$, e.g., if finite elements are used for computations. Our goal is to analyze the effect of the domain change on the optimal controls. More precisely, two new optimal control problems $\left(\mathrm{NP}_{h}\right)$ and $\left(\mathrm{DP}_{h}\right)$ in $\Omega_{h}$ are defined. The convergence of global or local solutions of problems $\left(\mathrm{NP}_{h}\right)$ and $\left(\mathrm{DP}_{h}\right)$ to the corresponding local or global solutions of (NP) and (DP), respectively, is investigated for the limit passage $h \rightarrow 0$. The error estimates for the difference of optimal controls obtained for both problems in an appropriate norm are derived in a function of the parameter $h$. We restrict ourselves to the case of a convex domain $\Omega \subset \mathbb{R}^{2}$ approximated by a polygonal domain $\Omega_{h} ; h$ is the maximal length of the edges of $\Omega_{h}$. A family of infinite dimensional control problems $\left(\mathrm{NP}_{h}\right)$ and $\left(\mathrm{DP}_{h}\right)$ defined in $\Omega_{h}$ is considered, and the solutions of $\left(\mathrm{NP}_{h}\right)$ and $\left(\mathrm{DP}_{h}\right)$ are compared with the solutions of (NP) and (DP), respectively. In this way, the influence of small changes in the domain on the solutions of the control problems is analyzed.

The numerical computation of the solution of (NP) and (DP) requires the discretization of the respective state equations, typically by using finite elements. If $\Omega$ is a polygonal domain, then it is covered by the union of the triangles of the mesh, and $\Gamma$

${ }^{*}$ Received by the editors June 9, 2009; accepted for publication (in revised form) December 30, 2009; published electronically March 10, 2010. This paper was prepared during a visit in Spring of 2009 of E. Casas at the Institut Elie Cartan, UMR 7502 Nancy-Université-CNRS-INRIA, Université Henri Poincaré Nancy 1.

http://www.siam.org/journals/sicon/48-6/76155.html

$\dagger$ †epartmento de Matemática Aplicada y Ciencias de la Computación, E.T.S.I. Industriales y de Telecomunicación, Universidad de Cantabria, 39005 Santander, Spain (eduardo.casas@unican.es). This author was partially supported by the Spanish Ministry of Science and Innovation under projects MTM2008-04206 and "Ingenio Mathematica (i-MATH)" CSD2006-00032 (Consolider Ingenio 2010).

${ }^{\ddagger}$ Institut Elie Cartan, UMR 7502 Nancy-Université-CNRS-INRIA, Laboratoire de Mathématiques, Université Henri Poincaré Nancy 1, B.P. 239, 54506 Vandoeuvre lés Nancy Cedex, France, and Systems Research Institute, Polish Academy of the Sciences, ul. Newelska 6, 01-447 Warszawa, Poland (Jan.Sokolowski@iecn.u-nancy.fr). 
remains invariable. Then problems (NP) and (DP) are approximated by some discrete problems, and it is possible to estimate the differences $\left\|\bar{u}-\bar{u}_{h}\right\|_{L^{2}(\Gamma)}$ between the different solutions of (NP) and (DP) and the corresponding discrete approximations; see [3] or [4] for the Neumann case and [5] for the Dirichlet case. In the problems that we are considering here, the situation is more complicated because the numerical analysis with finite elements requires the approximation of $\Omega$ by a new (typically polygonal) domain $\Omega_{h}$, so that the comparison between the solutions $\bar{u}$ and $\bar{u}_{h}$ is more involved because $\bar{u} \in L^{2}(\Gamma)$ and $\bar{u}_{h} \in L^{2}\left(\Gamma_{h}\right)$, where $\Gamma_{h}$ is the boundary of $\Omega_{h}$. This difficulty can be overcome by using convenient parametrizations of $\Gamma$ and $\Gamma_{h}$, but there are still some technical difficulties for the error analysis. In this paper we do not consider the numerical approximations of (NP) or (DP); we just analyze what happens if $\Omega$ is approximated by a polygonal domain $\Omega_{h}$, and (NP) and (DP) are transformed into two new infinite dimensional control problem $\left(\mathrm{NP}_{h}\right)$ and $\left(\mathrm{DP}_{h}\right)$.

In section 6 we prove that the order of the approximation for the Neumann control problem is $h^{5 / 3}$. This order has an interesting consequence. Indeed, to numerically solve a Neumann control problem, piecewise constant or piecewise linear functions are typically taken to approximate the controls. In both cases, the maximal order of the error estimates is $h$ or $h^{3 / 2}$, respectively; see [3]. A consequence of our estimate is that we also have error estimates of order $h$ or $h^{3 / 2}$, depending on the type of approximation used for the controls, for a fully discretized control problem using piecewise linear approximation of the states on a polygonal domain. Order $h^{3 / 2}$ is also obtained if we follow the procedure suggested by Hinze in [8], where no control discretization is considered; only the state and adjoint states are discretized.

For the Dirichlet control problem we prove in section 9 that the order of the approximation is $h$. This is better than the estimate derived in [5] for the numerical discretization of the control problem for polygonal domains. Order $h^{1-1 / p}$, with $2<p<+\infty$, was proved in [5]. There $p$ depends on the angles of $\Omega_{h}$ and $p \searrow 2$ when the angles of $\Omega_{h}$ approximate $\pi$; this is the case when $h \rightarrow 0$. Therefore, order $h^{1 / 2}$ can be deduced from our result in section 9 and the result of [5] for a full discretization of the control problem. However, in the linear-quadratic case a superconvergence result was recently obtained in [6] under some assumptions on the triangulation of $\Omega_{h}$. They obtained the order $h^{3 / 2}$ for the numerical approximation of Dirichlet control problems defined on curved domains, where no control discretization is considered. We believe that the order $h^{1 / 2}$ can be improved to order $h$ without restrictive assumptions on the triangulation. The low convergence order proved in [5] is a consequence of the low regularity of the optimal control due to the lack of the regularity of the polygonal boundary. For a smooth domain $\Omega$ we have more regularity of the optimal controls which can lead to better error estimates. This was the case in [6].

Though the analysis of the Dirichlet control problem follows the same steps given for the analysis of the Neumann case, the arguments are different, and it is not evident how to change the arguments; in fact, the results obtained are not the same for the Neumann and Dirichlet cases, respectively.

The plan of the paper is as follows. In section 2 we introduce the Neumann control problem, and we study the existence, uniqueness, and regularity of the state equation (2.1) as well as the existence of a solution for problem (NP). In section 3 the first and second order optimality conditions for (NP) are established, which are the essential tools for deriving the error estimates. The domains $\Omega_{h}, h>0$, are introduced in section 4. Additionally, in section 4 we define a one-to-one mapping $g_{h}: \Gamma_{h} \longrightarrow \Gamma$ that allows us to compare the solutions $\bar{u}$ of $(\mathrm{NP})$ and $\bar{u}_{h}$ of $\left(\mathrm{NP}_{h}\right)$ in the norm $\left\|\bar{u}-\bar{u}_{h} \circ g_{h}^{-1}\right\|_{L^{2}(\Gamma)}$. In section 5 we prove that problems $\left(\mathrm{NP}_{h}\right)$ realize a correct 
approximation of (NP) in the sense that global solutions of $\left(\mathrm{NP}_{h}\right)$ converge strongly to global solutions of (NP) and the strict local solutions of (NP) can be approximated by local solutions of problems $\left(\mathrm{NP}_{h}\right)$. A crucial result in this section is the derivation of the estimates for the differences of states and of adjoint states defined in $\Omega$ and $\Omega_{h}$, respectively. The reader is referred to Theorems 5.1 and 5.2 for the estimates in the spaces $H^{s}\left(\Omega_{h}\right)$, with $0 \leq s \leq 3 / 2$. One key point in this proof is the use of a modification of the Aubin-Nitsche argument to derive error estimates in the $L^{2}$ norm for finite element approximations. This approach used in the case of linear equations can be adapted to semilinear problems, as is shown. Finally, in section 6 we derive the error estimates for the controls and the corresponding states and adjoint states. In section 7 we define the Dirichlet control problem and we establish the first and second order optimality conditions. In this case, the second order sufficient optimality conditions are not so simple to get in the Neumann case because the cost functional is not of class $C^{2}$ in $L^{2}(\Gamma)$. Moreover, we improve the result given in [5] in the sense that we get that the feasible controls satisfying the sufficient optimality conditions are strict local minima of (DP) in the sense of the $L^{2}$-topology. In [5], the local optimality in the sense of the $L^{\infty}$-topology was proved. In section 8 we define the control problems $\left(\mathrm{DP}_{h}\right)$ and we prove that they properly approximate problem (DP). We finish by deriving the error estimates in section 9 .

2. Neumann control problem. Let us introduce the Neumann control problem

$$
(\mathrm{NP})\left\{\begin{array}{l}
\min J(u)=\int_{\Omega} L\left(x, y_{u}(x)\right) d x+\frac{N}{2} \int_{\Gamma} u^{2}(x) d \sigma(x) \\
\text { subject to }\left(y_{u}, u\right) \in\left(L^{\infty}(\Omega) \cap H^{1}(\Omega)\right) \times L^{2}(\Gamma) \\
\alpha \leq u(x) \leq \beta \quad \text { for a.e. } x \in \Gamma
\end{array}\right.
$$

where $\Gamma$ is a smooth manifold and $y_{u}$ is the state associated to the control $u$ given by a solution of the Neumann problem

$$
\left\{\begin{array}{rlll}
-\Delta y+a(x, y) & = & 0 & \text { in } \Omega \\
\partial_{\nu} y & = & u & \text { on } \Gamma
\end{array}\right.
$$

The following hypotheses are imposed on the data of problem (NP).

(N1) $\Omega$ is an open, convex, and bounded domain in $\mathbb{R}^{2}$, with the boundary $\Gamma$ of class $C^{2}$. Moreover, we assume that $N>0$ and $-\infty \leq \alpha<\beta \leq+\infty$.

(N2) $L: \Omega \times \mathbb{R} \longrightarrow \mathbb{R}$ and $a: \Omega \times \mathbb{R} \longrightarrow \mathbb{R}$ are Carathéodory functions of class $C^{2}$ with respect to the second variable, $L(\cdot, 0) \in L^{1}(\Omega), a(\cdot, 0) \in L^{\infty}(\Omega)$, and for every $M>0$ there exists a constant $C_{M}$ such that for almost all $x \in \Omega$ and all $|y|,\left|y_{i}\right| \leq M, i=1,2$, the following inequalities hold:

$$
\left\{\begin{array}{l}
\sum_{j=1}^{2}\left\{\left|\frac{\partial^{j} L}{\partial y^{j}}(x, y)\right|+\left|\frac{\partial^{j} a}{\partial y^{j}}(x, y)\right|\right\} \leq C_{M} \\
\left|\frac{\partial^{2} L}{\partial y^{2}}\left(x, y_{2}\right)-\frac{\partial^{2} L}{\partial y^{2}}\left(x, y_{1}\right)\right|+\left|\frac{\partial^{2} a}{\partial y^{2}}\left(x, y_{2}\right)-\frac{\partial^{2} a}{\partial y^{2}}\left(x, y_{1}\right)\right| \leq C_{M}\left|y_{2}-y_{1}\right|
\end{array}\right.
$$

Copyright (c) by SIAM. Unauthorized reproduction of this article is prohibited. 
We also assume

$$
\left\{\begin{array}{l}
\frac{\partial a}{\partial y}(x, y) \geq 0 \text { for a.a. } x \in \Omega \text { and } \forall y \in \mathbb{R} \\
\exists E \subset \Omega \text { and } \Lambda>0 \text { such that }|E|>0 \text { and } \frac{\partial a}{\partial y}(x, y) \geq \Lambda \forall(x, y) \in E \times \mathbb{R} .
\end{array}\right.
$$

We observe that, by our assumptions (N1) and (N2), for every $u \in L^{2}(\Gamma)$ the state equation (2.1) has a unique solution $y_{u} \in L^{\infty}(\Omega) \cap H^{1}(\Omega)$. The proof is standard, and some estimates can be derived:

$$
\left\|y_{u}\right\|_{H^{1}(\Omega)}+\|y\|_{L^{\infty}(\Omega)} \leq C_{E}\left(\|a(\cdot, 0)\|_{L^{2}(\Omega)}+\|u\|_{L^{2}(\Gamma)}\right) .
$$

Moreover, if $u \in H^{1 / 2}(\Gamma)$, then $y_{u} \in H^{2}(\Omega)$, and we have an analogous estimate with the $L^{2}(\Gamma)$-norm of $u$ replaced by the $H^{1 / 2}(\Gamma)$-norm.

To ensure the existence of a global optimal solution of problem (NP), we need an additional hypothesis.

(N3) Either $\alpha, \beta \in \mathbb{R}$ or the following assumption holds:

$$
L(x, y) \geq \psi_{L}(x)+\Lambda_{L} y^{2}, \text { with } \psi_{L} \in L^{1}(\Omega) \text { and } N+4 C_{E}^{2} \min \left\{0, \Lambda_{L}\right\}>0,
$$

where $C_{E}$ is as in (2.4).

Indeed, if we take a minimizing sequence $\left\{u_{k}\right\}_{k=1}^{\infty}$ of problem (NP), then either $\alpha, \beta \in \mathbb{R}$ and consequently $\left\{u_{k}\right\}_{k=1}^{\infty}$ is bounded in $L^{\infty}(\Gamma)$ or

$$
\begin{aligned}
J\left(u_{k}\right) & \geq \int_{\Omega} \psi_{L}(x) d x+\Lambda_{L} \int_{\Omega} y_{k}^{2}(x) d x+\frac{N}{2}\left\|u_{k}\right\|_{L^{2}(\Gamma)}^{2} \\
& \geq \int_{\Omega} \psi_{L}(x) d x+2 \min \left\{0, \Lambda_{L}\right\} C_{E}^{2}\left(\|a(\cdot, 0)\|_{L^{2}(\Omega)}^{2}+\left\|u_{k}\right\|_{L^{2}(\Gamma)}^{2}\right)+\frac{N}{2}\left\|u_{k}\right\|_{L^{2}(\Gamma)}^{2} \\
& =C+\left(\frac{N}{2}+2 \min \left\{0, \Lambda_{L}\right\} C_{E}^{2}\right)\left\|u_{k}\right\|_{L^{2}(\Gamma)}^{2},
\end{aligned}
$$

which allows us to conclude again that $\left\{u_{k}\right\}_{k=1}^{\infty}$ is bounded in $L^{2}(\Gamma)$. The remaining part of the proof is classical.

3. First and second order optimality conditions for (NP). In this section we establish the first and second order optimality conditions for the local minimum of (NP), which are necessary for deriving error estimates when approximating (NP) by $\left(\mathrm{NP}_{h}\right)$. Since problem $(\mathrm{NP})$ is not necessarily convex, it may have more than one global solution as well as some local solutions which are not global. The optimality system for a local solution is stated in the following theorem, where we also establish the regularity of the local minima.

TheOREm 3.1. Let $\bar{u}$ be a local minimum of $(\mathrm{NP})$. Then $\bar{u} \in C^{0,1}(\Gamma)$, and there exist elements $\bar{y}, \bar{\varphi} \in W^{2, p}(\Omega)$ for every $1 \leq p<+\infty$ such that

$$
\begin{aligned}
& \left\{\begin{array}{rlll}
-\Delta \bar{y}+a(x, \bar{y}) & = & 0 & \text { in } \Omega, \\
\partial_{\nu} \bar{y} & = & \bar{u} & \text { on } \Gamma,
\end{array}\right.
\end{aligned}
$$

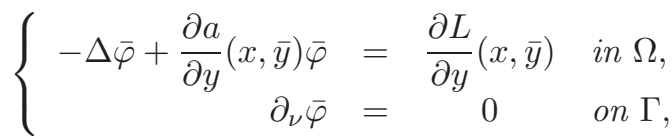

$$
\begin{aligned}
& \int_{\Gamma}(\bar{\varphi}(x)+N \bar{u}(x))(v(x)-\bar{u}(x)) d \sigma(x) \geq 0 \quad \forall \alpha \leq v \leq \beta .
\end{aligned}
$$

Copyright $@$ ㅇ by SIAM. Unauthorized reproduction of this article is prohibited. 
Sketch of the proof. First, we note that $J: L^{2}(\Gamma) \longrightarrow \mathbb{R}$ is of class $C^{1}$ (in fact, it is of class $C^{2}$ ) and

$$
J^{\prime}(\bar{u}) v=\int_{\Gamma}(\bar{\varphi}(x)+N \bar{u}(x)) v(x) d \sigma(x),
$$

where $\bar{\varphi} \in L^{\infty}(\Omega) \cap H^{1}(\Omega)$ is the solution of (3.2) and $\bar{y}$ is the state associated to $\bar{u}$ and consequently the unique solution of $(3.1)$ in $L^{\infty}(\Omega) \cap H^{1}(\Omega)$. The well-known optimality condition

$$
J^{\prime}(\bar{u})(v-\bar{u}) \geq 0 \quad \forall \alpha \leq v \leq \beta
$$

along with the expression of $J^{\prime}$ lead to (3.3). Now (3.3) implies

$$
\bar{u}(x)=\operatorname{Proj}_{[\alpha, \beta]}\left(-\frac{1}{N} \bar{\varphi}(x)\right)=\max \left\{\alpha, \min \left\{-\frac{1}{N} \bar{\varphi}(x), \beta\right\}\right\} .
$$

From our assumption (N2) and the boundedness of $\bar{y}$ we have that

$$
\frac{\partial L}{\partial y}(x, \bar{y}(x)), \frac{\partial a}{\partial y}(x, \bar{y}(x)) \in L^{\infty}(\Omega) .
$$

Therefore, we can use the elliptic regularity results (see Grisvard [7, Chapter 2]) to deduce that $\bar{\varphi} \in W^{2, p}(\Omega)$ for every $1 \leq p<+\infty$. Moreover, since $W^{2, p}(\Omega) \subset C^{1}(\bar{\Omega})$ for every $p>2$, we get from (3.4) that $\bar{u}$ is Lipschitz in $\Gamma$. Finally, from (3.1) and using again the elliptic regularity results, we conclude that $\bar{y} \in W^{2, p}(\Omega)$ for every $1 \leq p<+\infty$.

Let us observe that (3.3) is equivalent to $\bar{\varphi}+N \bar{u}=0$ on $\Gamma$ if $\alpha=-\infty$ and $\beta=+\infty$. In this case $\bar{u}=-\bar{\varphi} / N \in W^{2-1 / p, p}(\Gamma)$ for all $1 \leq p<+\infty$.

We finish this section by stating the second order optimality conditions. Given a local minimum $\bar{u}$, the associated cone of critical directions is defined by

$$
C_{\bar{u}}=\left\{v \in L^{2}(\Gamma) \text { satisfying (3.5) and such that } v(x)=0 \text { if }|\bar{\varphi}(x)+N \bar{u}(x)|>0\right\},
$$

$$
v(x)= \begin{cases}\geq 0 & \text { if } \bar{u}(x)=\alpha \\ \leq 0 & \text { if } \bar{u}(x)=\beta\end{cases}
$$

Then we have the following result.

THEOREM 3.2. If $\bar{u}$ is a local minimum of problem (NP), then $J^{\prime \prime}(\bar{u}) v^{2} \geq 0$ for all $v \in C_{\bar{u}}$. Reciprocally, if $\bar{u}$ is a feasible control for problem (NP) satisfying the first order optimality conditions (3.1)-(3.3) and the coercivity condition

$$
J^{\prime \prime}(\bar{u}) v^{2}>0 \quad \forall v \in C_{\bar{u}} \backslash\{0\},
$$

then there exist $\varepsilon>0$ and $\delta>0$ such that

$$
J(\bar{u})+\frac{\delta}{2}\|u-\bar{u}\|_{L^{2}(\Gamma)} \leq J(u)
$$

for every $\alpha \leq u \leq \beta$ such that $\|u-\bar{u}\|_{L^{2}(\Gamma)}<\varepsilon$.

For the details, the reader is referred to [2] and [4]. An important fact is that condition (3.6) holds if and only if

$$
\exists \mu>0 \text { and } \vartheta>0 \text { such that } J^{\prime \prime}(\bar{u}) v^{2} \geq \mu\|v\|_{L^{2}(\Gamma)}^{2} \forall v \in C_{\bar{u}}^{\vartheta},
$$

where

$$
C_{\bar{u}}^{\vartheta}=\left\{v \in L^{2}(\Gamma) \text { satisfying }(3.5) \text { and } v(x)=0 \text { if }|\bar{\varphi}(x)+N \bar{u}(x)|>\vartheta\right\} .
$$

Copyright ( by SIAM. Unauthorized reproduction of this article is prohibited. 


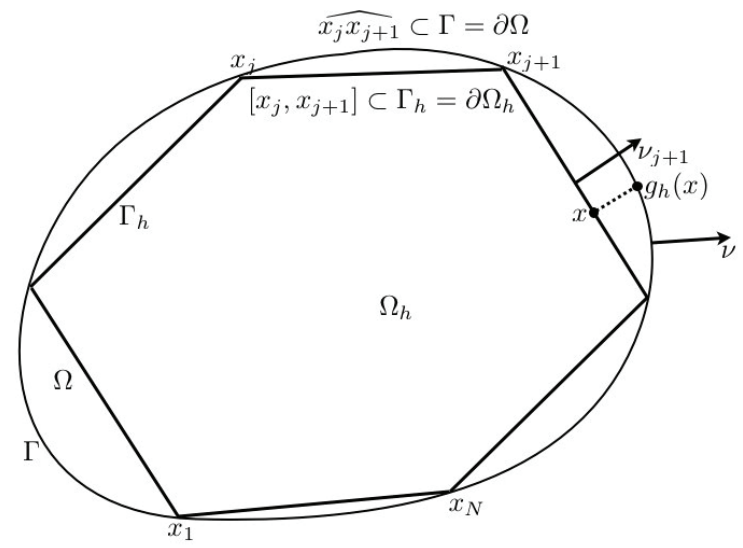

FIG. 4.1. Polygonal domain $\Omega_{h} \subset \Omega$ and its boundary $\Gamma_{h}$.

4. Control problem $\left(\mathrm{NP}_{\boldsymbol{h}}\right)$. In order to define the control problem $\left(\mathrm{NP}_{h}\right)$, we consider a polygonal approximation of $\Omega$. We fix a set of points $\left\{x_{j}\right\}_{j=1}^{N(h)} \subset \Gamma$, the nodes being ordered clockwise. We set

$$
h_{j}=\left|x_{j+1}-x_{j}\right|, \quad h=\max _{1 \leq j \leq N(h)} h_{j}, \quad \tau_{j}=\frac{1}{h_{j}}\left(x_{j+1}-x_{j}\right),
$$

where we denote $x_{N(h)+1}=x_{1} . \Gamma_{h}$ is the polygonal line defined by the nodes $\left\{x_{j}\right\}_{j=1}^{N(h)}$, and $\Omega_{h}$ is the polygon delimited by $\Gamma_{h}$; see Figure 4.1. Since $\Omega$ is convex, it is clear that $\Omega_{h} \subset \Omega$. For every $1 \leq j \leq N(h), \widehat{x_{j} x_{j+1}}$ denotes the arc of $\Gamma$ delimited by the points $x_{j}$ and $x_{j+1}$. Then we have that $\Gamma=\cup_{j=1}^{N(h)} \widehat{x_{j} x_{j+1}}$ and $\Gamma_{h}=\cup_{j=1}^{N(h)}\left[x_{j}, x_{j+1}\right]$. For every $1 \leq j \leq N(h), \nu_{j}$ represents the unit outward normal vector to $\Omega_{h}$ on the boundary edge $\left(x_{j}, x_{j+1}\right)$.

Now we introduce a parametrization of $\Gamma$ as follows:

$$
\psi_{j}:\left[0, h_{j}\right] \longrightarrow \widehat{x_{j} x_{j+1}} \subset \Gamma \text { is defined by } \psi_{j}(t)=x_{j}+t \tau_{j}+\phi_{j}(t) \nu_{j},
$$

where $\phi_{j}:\left[0, h_{j}\right] \longrightarrow[0,+\infty)$ is chosen such that $\psi_{j}(t) \in \Gamma$. It is evident that $\phi_{j}$ is uniquely defined. Since $\Omega$ is convex and $\Gamma$ is of class $C^{2}$, the following properties hold:

1. $\phi_{j}$ is of class $C^{2}$ and $\phi_{j}(0)=\phi_{j}\left(h_{j}\right)=0$.

2. There exists a constant $C_{\Gamma}>0$ such that $\phi_{j}(t)+h\left|\phi_{j}^{\prime}(t)\right| \leq C_{\Gamma} h_{j}^{2} \leq C_{\Gamma} h^{2}$ for all $t \in\left[0, h_{j}\right]$.

Finally, we define

$g_{h}: \Gamma_{h} \longrightarrow \Gamma,\left.\quad g_{h}\right|_{\left[x_{j}, x_{j+1}\right]}(x)=\left.g_{h}\right|_{\left[x_{j}, x_{j+1}\right]}\left(x_{j}+t \tau_{j}\right)=x_{j}+t \tau_{j}+\phi_{j}(t) \nu_{j}=\psi_{j}(t)$.

Clearly $g_{h}$ is one-to-one. We denote by $\nu(x)$ the unit outward normal vector to $\Gamma$ at the point $x$ and by $\tau(x)$ the unit tangent vector such that $\{\tau(x), \nu(x)\}$ is a direct reference system in $\mathbb{R}^{2}$. We can obtain the expressions for these vectors from the parametrization. If $x$ is a point of the arc $\widehat{x_{j} x_{j+1}}$, then

$$
\tau(x)=\frac{1}{\sqrt{1+\phi_{j}^{\prime}(t)^{2}}}\left(\tau_{j}+\phi_{j}^{\prime}(t) \nu_{j}\right) \text { and } \nu(x)=\frac{1}{\sqrt{1+\phi_{j}^{\prime}(t)^{2}}}\left(\nu_{j}-\phi_{j}^{\prime}(t) \tau_{j}\right) .
$$


From these expressions and the properties of $\phi_{j}$ we deduce that

$$
\left|\nu\left(g_{h}(x)\right)-\nu_{j}\right| \leq C_{\Gamma} h \sqrt{C_{\Gamma}^{2} h^{2}+1} \quad \forall x \in\left[x_{j}, x_{j+1}\right] ;
$$

the same inequality holds true for $\left|\tau\left(g_{h}(x)\right)-\tau_{j}\right|$. Since we are interested in the case of $h \rightarrow 0$, we can assume that $h<1$ and then

$$
\max \left\{\left|\tau\left(g_{h}(x)\right)-\tau_{h}(x)\right|,\left|\nu\left(g_{h}(x)\right)-\nu_{h}(x)\right|\right\} \leq\left(C_{\Gamma}^{2}+1\right) h \quad \forall x \in \Gamma_{h},
$$

where $\tau_{h}(x)=\tau_{j}$ and $\nu_{h}(x)=\nu_{j}$ if $x \in\left(x_{j}, x_{j+1}\right)$.

Given a function $v \in L^{1}(\Gamma)$, we have

$$
\int_{\Gamma} v(x) d \sigma(x)=\sum_{j=1}^{N(h)} \int_{0}^{h_{j}} v\left(\psi_{j}(t)\right) \sqrt{1+\phi_{j}^{\prime}(t)^{2}} d t
$$

and

$$
\int_{\Gamma_{h}} v\left(g_{h}(x)\right) d \sigma_{h}(x)=\sum_{j=1}^{N(h)} \int_{0}^{h_{j}} v\left(g_{h}\left(x_{j}+t \tau_{j}\right)\right) d t=\sum_{j=1}^{N(h)} \int_{0}^{h_{j}} v\left(\psi_{j}(t)\right) d t .
$$

From these expressions we deduce that

$$
\int_{\Gamma_{h}}\left|v\left(g_{h}(x)\right)\right| d \sigma_{h}(x) \leq \int_{\Gamma}|v(x)| d \sigma(x) \quad \forall v \in L^{1}(\Gamma)
$$

and

$$
\begin{array}{r}
\left|\int_{\Gamma} v(x) d \sigma(x)-\int_{\Gamma_{h}} v\left(g_{h}(x)\right) d \sigma_{h}(x)\right| \leq \sum_{j=1}^{N(h)} \int_{0}^{h_{j}}\left|v\left(\psi_{j}(t)\right)\right|\left|1-\sqrt{1+\phi_{j}^{\prime}(t)^{2}}\right| d t \\
\quad \leq C_{\Gamma} h^{2} \sum_{j=1}^{N(h)} \int_{0}^{h_{j}}\left|v\left(\psi_{j}(t)\right)\right| d t \leq C_{\Gamma} h^{2} \int_{\Gamma}|v(x)| d \sigma(x) \quad \forall v \in L^{1}(\Gamma) .
\end{array}
$$

We also have

$$
\int_{\Gamma} v(x) d \sigma(x)=\int_{\Gamma_{h}} v\left(g_{h}(x)\right)\left|D g_{h}(x) \cdot \tau_{h}(x)\right| d \sigma_{h}(x) \quad \forall v \in L^{1}(\Gamma) .
$$

In the domain $\Omega_{h}$ defined above we consider the state equation

$$
\left\{\begin{array}{rlll}
-\Delta y+a(x, y) & =0 & & \text { in } \Omega_{h} \\
\partial_{\nu_{h}} y & = & & \text { on } \Gamma_{h}
\end{array}\right.
$$

and the associated control problem

$$
\left(\mathrm{NP}_{h}\right)\left\{\begin{array}{l}
\min J_{h}(u)=\int_{\Omega_{h}} L\left(x, y_{h, u}(x)\right) d x+\frac{N}{2} \int_{\Gamma_{h}} u^{2}(x) d \sigma_{h}(x) \\
\text { subject to }\left(y_{h, u}, u\right) \in\left(L^{\infty}\left(\Omega_{h}\right) \cap H^{1}\left(\Omega_{h}\right)\right) \times L^{2}\left(\Gamma_{h}\right), \\
\alpha \leq u(x) \leq \beta \quad \text { for a.e. } x \in \Gamma_{h} .
\end{array}\right.
$$

Copyright $@$ ㅇ by SIAM. Unauthorized reproduction of this article is prohibited. 
Since we are interested in the behavior of the solutions of $\left(\mathrm{NP}_{h}\right)$ when $h \rightarrow 0$, we can assume without any loss of generality that there exists $h_{0}>0$ such that the set $E \subset \Omega$, introduced in assumption (N2), is also contained in $\Omega_{h}$ for every $h \leq h_{0}$. Then assumptions (N1) and (N2) imply the existence of a unique solution $y_{h, u}$ of (4.5) in $H^{1}\left(\Omega_{h}\right) \cap L^{\infty}\left(\Omega_{h}\right)$ for every $u \in L^{2}\left(\Gamma_{h}\right)$. Moreover, the inequality (2.4) can be rewritten as follows:

$$
\left\|y_{h, u}\right\|_{H^{1}\left(\Omega_{h}\right)}+\|y\|_{L^{\infty}\left(\Omega_{h}\right)} \leq C_{E}\left(\|a(\cdot, 0)\|_{L^{2}(\Omega)}+\|u\|_{L^{2}\left(\Gamma_{h}\right)}\right) \quad \forall h \leq h_{0} .
$$

Since $\Omega_{h}$ is a convex polygonal domain, we have that $y_{h, u} \in H^{2}\left(\Omega_{h}\right)$ whenever $u \in$ $H^{1 / 2}\left(\Gamma_{h}\right)$; see, for instance, Grisvard [7, Chapter 4].

Arguing as in section 2, we can prove that problem $\left(\mathrm{NP}_{h}\right)$ has at least one global minimum for every $h \leq h_{0}$. Furthermore, we have the optimality system analogous to $(3.1)-(3.3)$.

THEOREM 4.1. Let $\bar{u}_{h}$ be a local minimum of $\left(\mathrm{NP}_{h}\right)$. Then $\bar{u}_{h} \in H^{1}\left(\Gamma_{h}\right)$ and there exist elements $\bar{y}_{h}, \bar{\varphi}_{h} \in H^{2}\left(\Omega_{h}\right)$ such that

$$
\begin{aligned}
& \left\{\begin{array}{rlll}
-\Delta \bar{y}_{h}+a\left(x, \bar{y}_{h}\right) & = & & \text { in } \Omega_{h}, \\
\partial_{\nu} \bar{y}_{h} & = & \bar{u}_{h} & \text { on } \Gamma_{h},
\end{array}\right. \\
& \left\{\begin{array}{rccc}
-\Delta \bar{\varphi}_{h}+\frac{\partial a}{\partial y}\left(x, \bar{y}_{h}\right) \bar{\varphi}_{h} & = & \frac{\partial L}{\partial y}\left(x, \bar{y}_{h}\right) & \text { in } \Omega_{h}, \\
\partial_{\nu_{h}} \bar{\varphi}_{h} & = & 0 & \text { on } \Gamma_{h},
\end{array}\right. \\
& \int_{\Gamma_{h}}\left(\bar{\varphi}_{h}(x)+N \bar{u}_{h}(x)\right)\left(v_{h}(x)-\bar{u}_{h}(x)\right) d \sigma_{h}(x) \geq 0 \quad \forall \alpha \leq v_{h} \leq \beta .
\end{aligned}
$$

The proof of this theorem is the same as that of Theorem 3.1 with the only difference concerning the regularity of $\left(\bar{u}_{h}, \bar{y}_{h}, \bar{\varphi}_{h}\right)$. This difference is due to the lack of the regularity of $\Gamma_{h}$, which is not $C^{1,1}$, and thus the regularity results used in Theorem 3.1 are not valid. However, taking into account that $\Omega_{h}$ is convex, we can deduce that $\varphi_{h} \in H^{2}\left(\Omega_{h}\right)$; see Grisvard [7, Chapter 3]. Moreover, we have

$$
\left\|\bar{\varphi}_{h}\right\|_{H^{2}\left(\Omega_{h}\right)} \leq C\left(\left\|\frac{\partial a}{\partial y}\left(x, \bar{y}_{h}\right)\right\|_{L^{2}\left(\Omega_{h}\right)}+\left\|\frac{\partial L}{\partial y}\left(x, \bar{y}_{h}\right)\right\|_{L^{2}\left(\Omega_{h}\right)}\right),
$$

where $C$ is independent of $h$. Hence from (4.6) and assumption (N2) it follows that

$$
\left\|\bar{\varphi}_{h}\right\|_{H^{2}\left(\Omega_{h}\right)} \leq M_{\bar{u}_{h}}
$$

where $M_{\bar{u}_{h}}$ is a constant depending on $\left\|\bar{u}_{h}\right\|_{L^{2}\left(\Gamma_{h}\right)}$. Using (4.9) we get

$$
\bar{u}_{h}(x)=\operatorname{Proj}_{[\alpha, \beta]}\left(-\frac{1}{N} \bar{\varphi}_{h}(x)\right)=\max \left\{\alpha, \min \left\{-\frac{1}{N} \bar{\varphi}_{h}(x), \beta\right\}\right\},
$$

which implies that $\bar{u}_{h} \in H^{1}\left(\Gamma_{h}\right)$; hence $\bar{y}_{h} \in H^{2}\left(\Omega_{h}\right)$ and

$$
\left\|\bar{y}_{h}\right\|_{H^{2}\left(\Omega_{h}\right)}+\left\|\bar{u}_{h}\right\|_{H^{1}\left(\Gamma_{h}\right)} \leq K_{\bar{u}_{h}},
$$

where once again $K_{\bar{u}_{h}}$ is a constant depending only on $\left\|\bar{u}_{h}\right\|_{L^{2}\left(\Gamma_{h}\right)}$ and independent of $h$.

$$
\begin{aligned}
& \text { If }-\infty<\alpha<\beta<+\infty \text {, then } \\
& \qquad\left\|\bar{u}_{h}\right\|_{L^{2}\left(\Gamma_{h}\right)} \leq \max \{|\alpha|,|\beta|\}\left|\Gamma_{h}\right|^{1 / 2} \leq \max \{|\alpha|,|\beta|\}|\Gamma|^{1 / 2} .
\end{aligned}
$$

Copyright (C) by SIAM. Unauthorized reproduction of this article is prohibited. 
If $\alpha=-\infty$ or $\beta=+\infty$, then by (2.5) and the same argument as that used at the end of section 2 we get for all $u_{h} \in L^{2}\left(\Gamma_{h}\right)$ with $\alpha \leq u_{h} \leq \beta$

$$
C+\left(\frac{N}{2}+2 \min \left\{0, \Lambda_{L}\right\} C_{E}^{2}\right)\left\|\bar{u}_{h}\right\|_{L^{2}\left(\Gamma_{h}\right)}^{2} \leq J_{h}\left(\bar{u}_{h}\right) \leq J_{h}\left(u_{h}\right) .
$$

If $\bar{u}_{h}$ is a global solution of $\left(\mathrm{NP}_{h}\right)$, then we can take $u_{h} \equiv c_{\alpha, \beta}$, with a constant $\alpha<c_{\alpha, \beta}<\beta$, and deduce from the above inequality, in view of (4.6), the boundedness of $\left\{\left\|\bar{u}_{h}\right\|_{L^{2}\left(\Gamma_{h}\right)}\right\}_{h \leq h_{0}}$. In any case, by (4.10) and (4.12) there is a constant $K>0$ such that

$$
\left\|\bar{y}_{h}\right\|_{H^{2}\left(\Omega_{h}\right)}+\left\|\bar{\varphi}_{h}\right\|_{H^{2}\left(\Omega_{h}\right)}+\left\|\bar{u}_{h}\right\|_{H^{1}\left(\Gamma_{h}\right)} \leq K \quad \forall h \leq h_{0} .
$$

When $\left\{\bar{u}_{h}\right\}_{h \leq h_{0}}$ are just local minima of problems $\left(\mathrm{NP}_{h}\right)$, the inequality (4.13) remains valid for $-\infty<\alpha<\beta<+\infty$ or for a bounded sequence $\left\{J_{h}\left(\bar{u}_{h}\right)\right\}_{h \leq h_{0}}$, which is true provided $\left\{\left\|\bar{u}_{h}\right\|_{L^{2}\left(\Gamma_{h}\right)}\right\}_{h \leq h_{0}}$ is bounded (cf. (4.6)).

5. Convergence analysis. The goal of this section is to prove the convergence, in a sense to be defined later, of the solutions $\bar{u}_{h}$ of $\left(\mathrm{NP}_{h}\right)$ to the solutions $\bar{u}$ of (NP). We also analyze the approximation of local minima of (NP) by local minima of problems $\left(\mathrm{NP}_{h}\right)$. In order to carry out this analysis, first we compare the solutions of (2.1) and (4.5).

TheOREM 5.1. Let $u \in H^{1 / 2}(\Gamma)$ and $u_{h} \in L^{2}\left(\Gamma_{h}\right)$, with

$$
\max \left\{\|u\|_{L^{2}(\Gamma)},\left\|u_{h}\right\|_{L^{2}\left(\Gamma_{h}\right)}\right\} \leq M
$$

Let $y_{u} \in H^{2}(\Omega)$ and $y_{h, u_{h}} \in H^{3 / 2}\left(\Omega_{h}\right)$ be the corresponding solutions of (2.1) and (4.5), respectively. Then there exists a constant $C_{M}>0$ independent of $h$ such that for all $0 \leq s \leq \frac{3}{2}$ the following estimate holds:

$$
\left\|y_{u}-y_{h, u_{h}}\right\|_{H^{s}\left(\Omega_{h}\right)} \leq C_{M}\left(\left\|u-u_{h} \circ g_{h}^{-1}\right\|_{L^{2}(\Gamma)}+h^{\frac{6-2 s}{3}}\left[1+\|u\|_{H^{1 / 2}(\Gamma)}\right]\right) .
$$

Proof. Let us introduce the intermediate problem

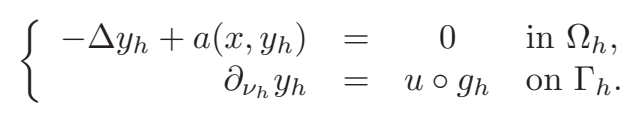

Then we have

$$
\left\|y_{u}-y_{h, u_{h}}\right\|_{H^{s}\left(\Omega_{h}\right)} \leq\left\|y_{u}-y_{h}\right\|_{H^{s}\left(\Omega_{h}\right)}+\left\|y_{h}-y_{h, u_{h}}\right\|_{H^{s}\left(\Omega_{h}\right)} .
$$

Let us estimate the second term of the right-hand side in (5.4). We set $\phi_{h}=y_{h}-y_{h, u_{h}}$. By subtraction of the equations satisfied by $y_{h}$ and $y_{h, u_{h}}$ and using the mean value theorem, we get

$$
\left\{\begin{array}{cccc}
-\Delta \phi_{h}+\frac{\partial a}{\partial y}\left(x, w_{h}\right) \phi_{h} & = & 0 & \text { in } \Omega_{h}, \\
\partial_{\nu_{h}} \phi_{h} & =u \circ g_{h}-u_{h} & & \text { on } \Gamma_{h},
\end{array}\right.
$$

where $w_{h}=y_{h}+\theta_{h}\left(y_{h, u_{h}}-y_{h}\right)$ and $0<\theta_{h}<1$. From (5.5) and assumption (2.3) it follows that

$$
\left\|\phi_{h}\right\|_{H^{1}\left(\Omega_{h}\right)}+\left\|\phi_{h}\right\|_{L^{\infty}\left(\Omega_{h}\right)} \leq\left\|u \circ g_{h}-u_{h}\right\|_{L^{2}\left(\Gamma_{h}\right)} .
$$

Copyright (c) by SIAM. Unauthorized reproduction of this article is prohibited. 
In view of (5.1), we can apply (2.4) and (4.6) to obtain that

$$
\left\|\frac{\partial a}{\partial y}\left(x, w_{h}\right)\right\|_{L^{\infty}\left(\Omega_{h}\right)} \leq C_{1}
$$

for some constant $C_{1}$ depending on $M$ (cf. assumption (N2)). Then we get

$$
\begin{array}{r}
\left\|\phi_{h}\right\|_{H^{3 / 2}\left(\Omega_{h}\right)} \leq C_{2}\left(\left\|\Delta \phi_{h}\right\|_{L^{2}\left(\Omega_{h}\right)}+\left\|u \circ g_{h}-u_{h}\right\|_{L^{2}\left(\Gamma_{h}\right)}\right) \\
\leq C_{2}\left(C_{1}\left\|\phi_{h}\right\|_{L^{2}\left(\Omega_{h}\right)}+\left\|u \circ g_{h}-u_{h}\right\|_{L^{2}\left(\Gamma_{h}\right)}\right) \leq C_{3}\left\|u \circ g_{h}-u_{h}\right\|_{L^{2}\left(\Gamma_{h}\right)} ;
\end{array}
$$

see [10, page 121] for the first estimate. Now (4.2) combined with the above inequality leads to

$$
\left\|y_{h}-y_{h, u_{h}}\right\|_{H^{s}\left(\Omega_{h}\right)} \leq C_{3}\left\|u-u_{h} \circ g_{h}^{-1}\right\|_{L^{2}(\Gamma)} \forall 0 \leq s \leq \frac{3}{2} .
$$

The remaining part of the proof is dedicated to the derivation of the inequality

$$
\left\|y_{u}-y_{h}\right\|_{H^{s}\left(\Omega_{h}\right)} \leq C h^{\frac{6-2 s}{3}}\left[\|u\|_{H^{1 / 2}(\Gamma)}+1\right] \forall 0 \leq s \leq \frac{3}{2},
$$

where $C$ depends on the constant $M$ given in (5.1). Thus (5.6) and (5.7) imply (5.2). The proof follows some steps. First, we consider the case $s=3 / 2$. Then by using the Aubin-Nitsche duality method we deduce the estimate for $s=0$. Finally, an appropriate interpolation inequality completes the proof.

Case 1: $s=3 / 2$. Let us use again the letter $\phi_{h}$ to denote $\phi_{h}=y_{u}-y_{h}$. By subtraction of the equations satisfied by $y_{u}$ and $y_{h}$ and by an application of the mean value theorem we get

$$
\left\{\begin{array}{cccc}
-\Delta \phi_{h}+\frac{\partial a}{\partial y}\left(x, w_{h}\right) \phi_{h} & = & 0 & \\
\partial_{\nu_{h}} \phi_{h} & = & \partial_{\nu_{h}} y_{u}-u \circ g_{h} & \text { on } \Gamma_{h} .
\end{array}\right.
$$

Using [10] once again, we get

$$
\begin{aligned}
\left\|\phi_{h}\right\|_{H^{3 / 2}\left(\Omega_{h}\right) \leq} & C_{3}\left\|\partial_{\nu_{h}} y_{u}-u \circ g_{h}\right\|_{L^{2}\left(\Gamma_{h}\right)} \\
\leq & C_{3}\left\{\left\|\nabla y_{u} \cdot \nu_{h}-\left(\nabla y_{u} \circ g_{h}\right) \cdot \nu_{h}\right\|_{L^{2}\left(\Gamma_{h}\right)}\right. \\
& \left.+\left\|\left(\nabla y_{u} \circ g_{h}\right) \cdot \nu_{h}-\left(\nabla y_{u} \circ g_{h}\right) \cdot\left(\nu \circ g_{h}\right)\right\|_{L^{2}\left(\Gamma_{h}\right)}\right\} \\
\leq & C_{3}\left\{\left\|\nabla y_{u}-\nabla y_{u} \circ g_{h}\right\|_{L^{2}\left(\Gamma_{h}\right)}+\left\|\nabla y_{u} \circ g_{h}\right\|_{L^{2}\left(\Gamma_{h}\right)}\left\|\nu_{h}-\nu \circ g_{h}\right\|_{L^{2}\left(\Gamma_{h}\right)}\right\} .
\end{aligned}
$$

From [1, Lemma 1] we have

$$
\left\|w-w \circ g_{h}\right\|_{L^{2}\left(\Gamma_{h}\right)} \leq C h^{r}\|w\|_{H^{r}(\Omega)} \forall 1 \leq r \leq 2 .
$$

Using this inequality with $r=1$ and $w=\nabla y$ in the above estimate for $\phi_{h}$ along with (4.1), we get

$$
\left\|y_{u}-y_{h}\right\|_{H^{3 / 2}\left(\Omega_{h}\right)} \leq C_{4} h\left\|y_{u}\right\|_{H^{2}(\Omega)} \leq C_{5} h\left[\|u\|_{H^{1 / 2}(\Gamma)}+1\right],
$$

where $C_{5}$ depends on the $L^{2}(\Omega)$-norm of $\frac{\partial a}{\partial y}\left(x, y_{u}\right) y_{u}$. By using (2.4) and assumption (N2) we get that the norm can be estimated by a constant depending on $M$, which implies that $C_{5}$ depends on $M$ as well.

Copyright $@$ by SIAM. Unauthorized reproduction of this article is prohibited. 
Case 2: $s=0$. Let us define the function $\mu_{h} \in L^{\infty}\left(\Omega_{h}\right)$ by

$$
\mu_{h}(x)=\left\{\begin{array}{cl}
\frac{a\left(x, y_{u}(x)\right)-a\left(x, y_{h}(x)\right)}{y_{u}(x)-y_{h}(x)} & \text { if } y_{u}(x) \neq y_{h}(x), \\
\Lambda & \text { otherwise; }
\end{array}\right.
$$

see (2.3) for the definition of $\Lambda$. Observe that $\mu_{h} \geq \Lambda>0$ in $E \subset \Omega_{h}$. Let $f \in L^{2}\left(\Omega_{h}\right)$ be arbitrary. We extend $f$ and $\mu_{h}$ to $\Omega$ by zero, and we define $z \in H^{2}(\Omega)$ and $z_{h} \in H^{2}\left(\Omega_{h}\right)$ as the solutions of the problems

$$
\left\{\begin{array}{rlll}
-\Delta z+\mu_{h}(x) z & = & f & \text { in } \Omega \\
\partial_{\nu} z & = & 0 & \text { on } \Gamma
\end{array}\right.
$$

and

$$
\left\{\begin{array}{rll}
-\Delta z_{h}+\mu_{h}(x) z_{h} & =f & \text { in } \Omega_{h} \\
\partial_{\nu_{h}} z_{h} & =0 & \text { on } \Gamma_{h}
\end{array}\right.
$$

Taking the difference of (5.11) and (5.12) and arguing as above, we get

$$
\begin{aligned}
\left\|z-z_{h}\right\|_{H^{3 / 2}\left(\Omega_{h}\right)} & \leq C_{6}\left\|\partial_{\nu_{h}} z\right\|_{L^{2}\left(\Gamma_{h}\right)}=C_{6}\left\|\nabla z \cdot \nu_{h}-\left(\nabla z \circ g_{h}\right) \cdot\left(\nu \circ g_{h}\right)\right\|_{L^{2}\left(\Gamma_{h}\right)} \\
& \leq C_{6}\left\{\left\|\nabla z-\nabla z \circ g_{h}\right\|_{L^{2}\left(\Gamma_{h}\right)}+\left\|\nabla z \circ g_{h}\right\|_{L^{2}\left(\Gamma_{h}\right)}\left\|\nu_{h}-\nu \circ g_{h}\right\|_{L^{2}\left(\Gamma_{h}\right)}\right\} \\
& \leq C_{7} h\|z\|_{H^{2}(\Omega)} \leq C_{8} h\|f\|_{L^{2}\left(\Omega_{h}\right)} .
\end{aligned}
$$

Now multiplying (5.12) by $y_{u}-y_{h}$, integrating by parts, and using the equations satisfied by $y_{u}$ and $y_{h}$, we get

$$
\begin{array}{r}
\int_{\Omega_{h}} f\left(y_{u}-y_{h}\right) d x=\int_{\Omega_{h}}\left\{\nabla z_{h}\left(\nabla y_{u}-\nabla y_{h}\right)+\left[a\left(x, y_{u}\right)-a\left(x, y_{h}\right)\right] z_{h}\right\} d x \\
=\int_{\Omega_{h}}\left\{\left(\nabla z_{h}-\nabla z\right)\left(\nabla y_{u}-\nabla y_{h}\right)+\left[a\left(x, y_{u}\right)-a\left(x, y_{h}\right)\right]\left(z_{h}-z\right)\right\} d x \\
\quad+\int_{\Omega_{h}}\left\{\nabla z \nabla y_{u}+a\left(x, y_{u}\right) z\right\} d x-\int_{\Omega_{h}}\left\{\nabla z \nabla y_{h}+a\left(x, y_{h}\right) z\right\} d x \\
\leq\left\|z_{h}-z\right\|_{H^{1}\left(\Omega_{h}\right)}\left\|y_{u}-y_{h}\right\|_{H^{1}\left(\Omega_{h}\right)}-\int_{\Omega \backslash \Omega_{h}}\left\{\nabla z \nabla y_{u}+a\left(x, y_{u}\right) z\right\} d x \\
+\int_{\Gamma} u z d \sigma-\int_{\Gamma_{h}}\left(u \circ g_{h}\right) z d \sigma_{h} .
\end{array}
$$

From (5.10) and (5.13) we obtain

$$
\left\|z_{h}-z\right\|_{H^{1}\left(\Omega_{h}\right)}\left\|y_{u}-y_{h}\right\|_{H^{1}\left(\Omega_{h}\right)} \leq C_{5} C_{8} h^{2}\left[\|u\|_{H^{1 / 2}(\Gamma)}+1\right]\|f\|_{L^{2}\left(\Omega_{h}\right)} .
$$

To estimate the second term on the right-hand side of (5.14), we use the inequality (see [1, Lemma 2])

$$
\|w\|_{L^{2}\left(\Omega \backslash \Omega_{h}\right)} \leq C h\|w\|_{H^{1}(\Omega)} .
$$

On the other hand, recalling that $0 \leq \phi_{j}(t) \leq C_{\Gamma} h^{2}$ for every $1 \leq j \leq N(h)$, we get the well-known estimate

$$
\left|\Omega \backslash \Omega_{h}\right| \leq C h^{2}
$$

Copyright $@$ ㅇ by SIAM. Unauthorized reproduction of this article is prohibited. 
From (5.16) and (5.17) we get

$$
\begin{aligned}
& \left|\int_{\Omega \backslash \Omega_{h}}\left\{\nabla z \nabla y_{u}+a\left(x, y_{u}\right) z\right\} d x\right| \\
\leq & \|\nabla z\|_{L^{2}\left(\Omega \backslash \Omega_{h}\right)}\left\|\nabla y_{u}\right\|_{L^{2}\left(\Omega \backslash \Omega_{h}\right)}+\left\|a\left(x, y_{u}\right)\right\|_{L^{2}\left(\Omega \backslash \Omega_{h}\right)}\|z\|_{L^{2}\left(\Omega \backslash \Omega_{h}\right)} \\
\leq & C h^{2}\|z\|_{H^{2}(\Omega)}\left\|y_{u}\right\|_{H^{2}(\Omega)}+\sqrt{\left|\Omega \backslash \Omega_{h}\right|}\left\|a\left(x, y_{u}\right)\right\|_{L^{\infty}(\Omega)} C h\|z\|_{H^{1}(\Omega)} \\
\leq & C_{9} h^{2}\left[\left\|y_{u}\right\|_{H^{2}(\Omega)}+1\right]\|f\|_{L^{2}\left(\Omega_{h}\right)} \leq C_{10} h^{2}\left[\|u\|_{H^{1 / 2}(\Gamma)}+1\right]\|f\|_{L^{2}\left(\Omega_{h}\right)},
\end{aligned}
$$

where $C_{10}$ depends on the constant $M$ given by (5.1).

Finally, we estimate the last term of (5.14) by using (4.2), (4.3), (5.1), and (5.9):

$$
\begin{aligned}
& \left|\int_{\Gamma} u z d \sigma-\int_{\Gamma_{h}}\left(u \circ g_{h}\right) z d \sigma_{h}\right| \\
\leq & \int_{\Gamma_{h}}\left|\left(u \circ g_{h}\right)\left(z \circ g_{h}-z\right)\right| d \sigma_{h}+C_{\Gamma} h^{2} \int_{\Gamma}|u z| d \sigma \\
\leq & \left\|u \circ g_{h}\right\|_{L^{2}\left(\Gamma_{h}\right)}\left\|z \circ g_{h}-z\right\|_{L^{2}\left(\Gamma_{h}\right)}+C_{\Gamma} h^{2}\|u\|_{L^{2}(\Gamma)}\|z\|_{L^{2}(\Gamma)} \\
\leq & C_{11} h^{2}\|u\|_{L^{2}(\Gamma)}\|z\|_{H^{2}(\Omega)} \leq C_{12} M h^{2}\|f\|_{L^{2}\left(\Omega_{h}\right)} .
\end{aligned}
$$

Now, from (5.14), (5.15), (5.18), and (5.19), we deduce

$$
\left\|y_{u}-y_{h}\right\|_{L^{2}\left(\Omega_{h}\right)} \leq C h^{2}\left[\|u\|_{H^{1 / 2}(\Gamma)}+1\right],
$$

where $C$ depends on $M$ but is independent of $h$.

Case 3: $0<s<3 / 2$. This case can be obtained from Case 1 combined with Case 2 and the interpolation inequality

$$
\|w\|_{H^{s}\left(\Omega_{h}\right)} \leq \varepsilon\|w\|_{H^{3 / 2}\left(\Omega_{h}\right)}+K \varepsilon^{-\frac{2 s}{3-2 s}}\|w\|_{L^{2}\left(\Omega_{h}\right)},
$$

which holds for any $\varepsilon>0$; see [7, Theorem 1.4.3.3]. By setting $\varepsilon=h^{(3-2 s) / 3}$ in (5.21) and using (5.10) and (5.20), we deduce (5.7).

The next step in our analysis is comparing the adjoint state equations corresponding to $y_{u}$ and $y_{h, u_{h}}$. More precisely, we introduce the adjoint states $\varphi_{u} \in H^{2}(\Omega)$ and $\varphi_{h, u_{h}} \in H^{2}\left(\Omega_{h}\right)$ as the solutions of the equations

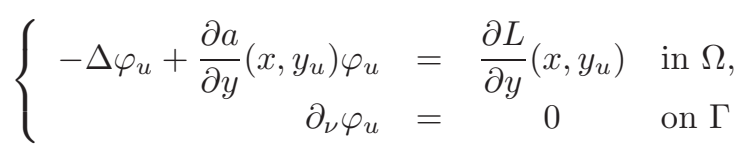

and

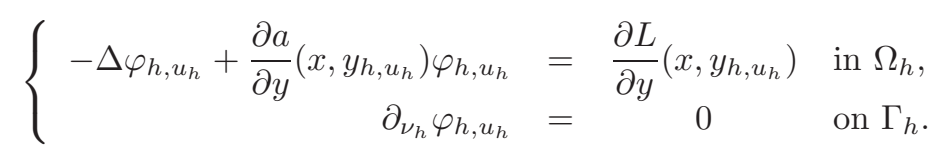

Then we have the following estimates.

TheOrem 5.2. Let $\left(u, y_{u}\right)$ and $\left(u_{h}, y_{h, u_{h}}\right)$ be as in Theorem 5.1. Let $\varphi_{u} \in H^{2}(\Omega)$ and $\varphi_{h, u_{h}} \in H^{2}\left(\Omega_{h}\right)$ be the corresponding solutions of (5.22) and (5.23), respectively. Then there exists a constant $C_{M}>0$ independent of $h$ such that for all $0 \leq s \leq \frac{3}{2}$ the following estimate holds:

$$
\left\|\varphi_{u}-\varphi_{h, u_{h}}\right\|_{H^{s}\left(\Omega_{h}\right)} \leq C_{M}\left(\left\|u-u_{h} \circ g_{h}^{-1}\right\|_{L^{2}(\Gamma)}+h^{\frac{6-2 s}{3}}\left[1+\|u\|_{H^{1 / 2}(\Gamma)}\right]\right) .
$$

Copyright (c) by SIAM. Unauthorized reproduction of this article is prohibited. 
Proof. We follow the steps of the proof of Theorem 5.1, with some simplifications, because now the equations are linear and the boundary conditions are homogeneous. To estimate $\varphi_{u}-\varphi_{h, u_{h}}$ we use estimates (5.2). Let us consider $\varphi_{h} \in H^{2}\left(\Omega_{h}\right)$ given by a solution of

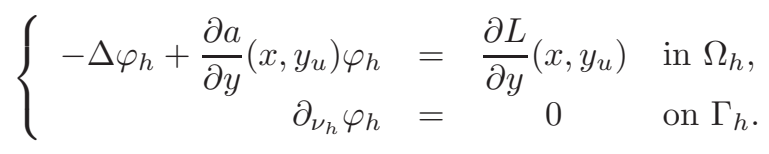

From assumption (N2) and estimates (5.2) we deduce the existence of a constant $C_{1}>0$ depending on $M$ such that

$$
\begin{gathered}
\left\|\frac{\partial L}{\partial y}\left(x, y_{u}\right)-\frac{\partial L}{\partial y}\left(x, y_{h, u_{h}}\right)\right\|_{L^{2}\left(\Omega_{h}\right)}+\left\|\left[\frac{\partial a}{\partial y}\left(x, y_{u}\right)-\frac{\partial a}{\partial y}\left(x, y_{h, u_{h}}\right)\right] \varphi_{h, u_{h}}\right\|_{L^{2}\left(\Omega_{h}\right)} \\
(5.26) \leq C_{1}\left\|y_{u}-y_{h, u_{h}}\right\|_{L^{2}\left(\Omega_{h}\right)} \leq C_{2}\left(\left\|u-u_{h} \circ g_{h}^{-1}\right\|_{L^{2}(\Gamma)}+h^{2}\left[1+\|u\|_{H^{1 / 2}(\Gamma)}\right]\right) .
\end{gathered}
$$

From (5.23), (5.25), and (5.26) we obtain

$$
\begin{aligned}
& \left\|\varphi_{h}-\varphi_{h, u_{h}}\right\|_{H^{3 / 2}\left(\Omega_{h}\right)} \leq C_{3}\left\|\Delta\left(\varphi_{h}-\varphi_{h, u_{h}}\right)\right\|_{L^{2}\left(\Omega_{h}\right)} \\
& \leq C_{4}\left(\left\|u-u_{h} \circ g_{h}^{-1}\right\|_{L^{2}(\Gamma)}+h^{2}\left[1+\|u\|_{H^{1 / 2}(\Gamma)}\right]\right) .
\end{aligned}
$$

The remaining part of the proof is devoted to the derivation of the estimate

$$
\left\|\varphi_{u}-\varphi_{h}\right\|_{H^{s}\left(\Omega_{h}\right)} \leq C h^{\frac{6-2 s}{3}},
$$

since (5.27) and (5.28) imply (5.24).

We start with the case of $s=3 / 2$. To this end, we define $\phi_{h}=\varphi_{u}-\varphi_{h}$. From (5.22) and (5.25) we get

$$
\left\{\begin{array}{rlcl}
-\Delta \phi_{h}+\frac{\partial a}{\partial y}\left(x, y_{u}\right) \phi_{h} & = & 0 & \text { in } \Omega_{h}, \\
\partial_{\nu_{h}} \phi_{h} & = & \partial_{\nu_{h}} \varphi_{u} & \text { on } \Gamma_{h} .
\end{array}\right.
$$

Then we have

$$
\left\|\varphi_{u}-\varphi_{h}\right\|_{H^{3 / 2}\left(\Omega_{h}\right)}=\left\|\phi_{h}\right\|_{H^{3 / 2}\left(\Omega_{h}\right)} \leq C_{1}\left\|\partial_{\nu_{h}} \varphi_{u}\right\|_{L^{2}\left(\Gamma_{h}\right)} \leq C_{2} h,
$$

where the estimate for $\partial_{\nu_{h}} \varphi_{u}$ is obtained in the same way as for $\partial_{\nu_{h}} z$ in (5.13).

Now, we prove (5.28) for $s=0$. To apply the Aubin-Nitsche duality method we define for every $f \in L^{2}(\Omega)$ vanishing in $\Omega \backslash \Omega_{h}$ the functions $z \in H^{2}(\Omega)$ and $z_{h} \in H^{2}\left(\Omega_{h}\right)$ given by solutions of the problems

$$
\left\{\begin{array}{rlll}
-\Delta z+\frac{\partial a}{\partial y}\left(x, y_{u}\right) z & = & \text { in } \Omega \\
\partial_{\nu} z & = & 0 & \text { on } \Gamma
\end{array}\right.
$$

and

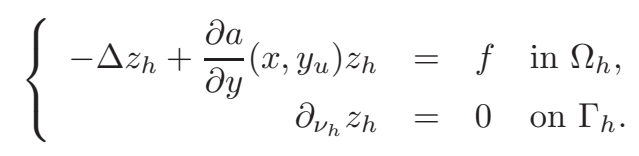

Copyright $@$ by SIAM. Unauthorized reproduction of this article is prohibited. 
As in (5.13), we get

$$
\left\|z-z_{h}\right\|_{H^{3 / 2}\left(\Omega_{h}\right)} \leq C h\|f\|_{L^{2}\left(\Omega_{h}\right)} .
$$

The same arguments as in the proof of Theorem 5.1, in view of (5.30) and (5.33), lead to

$$
\left\|\varphi_{u}-\varphi_{h}\right\|_{L^{2}\left(\Omega_{h}\right)} \leq C h^{2}
$$

where $C$ depends on $M$.

Finally, (5.28) is proved for $0<s<3 / 2$ in the same way as in Theorem 5.1, using the inequality (5.21) with $\varepsilon=h^{(3-2 s) / 3}$ along with inequalities (5.30) and (5.34).

COROLLARY 5.3. Under the assumptions of Theorem 5.2 the following inequality holds:

$$
\left\|\varphi_{u}-\varphi_{h, u_{h}}\right\|_{L^{2}\left(\Gamma_{h}\right)} \leq C_{M}\left(\left\|u-u_{h} \circ g_{h}^{-1}\right\|_{L^{2}(\Gamma)}+h^{5 / 3}\left[1+\|u\|_{H^{1 / 2}(\Gamma)}\right]\right)
$$

for a constant $C_{M}$ depending on $M$ but independent of $h$.

Proof. Using the function $\varphi_{h}$ defined in (5.25) and inequality (5.27), we get

$$
\begin{array}{r}
\left\|\varphi_{u}-\varphi_{h, u_{h}}\right\|_{L^{2}\left(\Gamma_{h}\right)} \leq\left\|\varphi_{u}-\varphi_{h}\right\|_{L^{2}\left(\Gamma_{h}\right)}+\left\|\varphi_{h}-\varphi_{h, u_{h}}\right\|_{L^{2}\left(\Gamma_{h}\right)} \\
\leq\left\|\varphi_{u}-\varphi_{h}\right\|_{L^{2}\left(\Gamma_{h}\right)}+C\left(\left\|u-u_{h} \circ g_{h}^{-1}\right\|_{L^{2}(\Gamma)}+h^{2}\left[1+\|u\|_{H^{1 / 2}(\Gamma)}\right]\right) .
\end{array}
$$

According to [7, Theorem 1.5.1.10], we have

$$
\left\|\varphi_{u}-\varphi_{h}\right\|_{L^{2}\left(\Gamma_{h}\right)}^{2} \leq K\left\{\varepsilon^{1 / 2}\left\|\nabla\left(\varphi_{u}-\varphi_{h}\right)\right\|_{L^{2}\left(\Omega_{h}\right)}^{2}+\varepsilon^{-1 / 2}\left\|\varphi_{u}-\varphi_{h}\right\|_{L^{2}\left(\Omega_{h}\right)}^{2}\right\} .
$$

Taking $s=1$ in (5.28) and $\varepsilon=h^{4 / 3}$ in (5.37) it follows that

$$
\left\|\varphi_{u}-\varphi_{h}\right\|_{L^{2}\left(\Gamma_{h}\right)} \leq C h^{5 / 3} .
$$

Finally, (5.36) and (5.38) lead to (5.35).

The remaining part of this section is devoted to the study of the convergence of solutions of $\left(\mathrm{NP}_{h}\right)$ to the solutions of (NP) with $h \rightarrow 0$. First, we prove that the solutions of $\left(\mathrm{NP}_{h}\right)$ converge to solutions of $(\mathrm{NP})$. Since $(\mathrm{NP})$ is not convex, we are also interested in an inverse property: if a given local minimum of (NP) can be approximated by local minima of problems $\left(\mathrm{NP}_{h}\right)$. This question is positively answered in this section. Let us start with a theorem which provides a precise meaning for the convergence of controls. We recall that problems $\left(\mathrm{NP}_{h}\right)$ admit at least one solution for each $h$ small enough (see the comments before Theorem 4.1).

TheOREM 5.4. Let $\bar{u}_{h}$ be a solution of problem $\left(\mathrm{NP}_{h}\right)$ for $h \leq h_{0}$. Then $\left\{\bar{u}_{h} \circ\right.$ $\left.g_{h}^{-1}\right\}_{0<h \leq h_{0}}$ is a bounded family in $H^{1}(\Gamma)$. If $\bar{u}$ is a weak limit for a subsequence, denoted in the same way, i.e., $\bar{u}_{h} \circ g_{h}^{-1} \rightarrow \bar{u}$ weakly in $H^{1}(\Gamma)$ with $h \rightarrow 0$, then $\bar{u}$ is a solution of problem (NP). Moreover,

$$
\lim _{h \rightarrow 0}\left\|\bar{y}-\bar{y}_{h}\right\|_{H^{3 / 2}\left(\Omega_{h}\right)}=0 \quad \text { and } \quad \lim _{h \rightarrow 0} J_{h}\left(\bar{u}_{h}\right) \rightarrow J(\bar{u}),
$$

where $\bar{y}$ and $\bar{y}_{h}$ denote the solutions of (2.1) and (4.5) corresponding to $\bar{u}$ and $\bar{u}_{h}$, respectively.

Proof. The boundedness of $\left\{\bar{u}_{h} \circ g_{h}^{-1}\right\}_{0<h<h_{0}}$ in $H^{1}(\Gamma)$ is an immediate consequence of (4.13). Let us prove the convergence of $\left\{J_{h}\left(\bar{u}_{h}\right)\right\}$. We denote by $\bar{y}_{h}$ and 
$\bar{y}$ the states associated to $\bar{u}_{h}$ and $\bar{u}$, respectively. Once again, (4.13) implies that $\left\|\bar{u}_{h}\right\|_{L^{2}\left(\Gamma_{h}\right)} \leq K$ for every $h \leq h_{0}$. Then we can use the estimates (5.2) with $s=3 / 2$ to get for $h \rightarrow 0$

$$
\left\|\bar{y}-\bar{y}_{h}\right\|_{H^{3 / 2}\left(\Omega_{h}\right)} \leq C_{M}\left(\left\|\bar{u}-\bar{u}_{h} \circ g_{h}^{-1}\right\|_{L^{2}(\Gamma)}+h\left[1+\|u\|_{H^{1 / 2}(\Gamma)}\right]\right) \rightarrow 0 .
$$

This convergence implies also that $\left\|\bar{y}-\bar{y}_{h}\right\|_{C(\bar{\Omega})} \rightarrow 0$. Then we have by assumption (N2) that

$$
\left|\int_{\Omega_{h}} L(x, \bar{y}(x)) d x-\int_{\Omega_{h}} L\left(x, \bar{y}_{h}(x)\right) d x\right| \leq C_{1} \int_{\Omega_{h}}\left|\bar{y}(x)-\bar{y}_{h}(x)\right| d x \rightarrow 0 .
$$

On the other hand, it is obvious that

$$
\lim _{h \rightarrow 0} \int_{\Omega \backslash \Omega_{h}} L(x, \bar{y}(x)) d x=0 .
$$

Finally, from (4.3) and by the strong convergence $\bar{u}_{h} \circ g_{h}^{-1} \rightarrow \bar{u}$ in $L^{2}(\Gamma)$ we obtain

$$
\begin{aligned}
& \left|\int_{\Gamma} \bar{u}^{2}(x) d \sigma(x)-\int_{\Gamma_{h}} \bar{u}_{h}^{2}(x) d \sigma_{h}(x)\right| \\
\leq & \left|\int_{\Gamma} \bar{u}^{2}(x) d \sigma(x)-\int_{\Gamma} \bar{u}_{h}^{2}\left(g_{h}^{-1}(x)\right) d \sigma(x)\right|+C h^{2} \rightarrow 0 .
\end{aligned}
$$

Collecting all these estimates, we deduce the convergence $J_{h}\left(\bar{u}_{h}\right) \rightarrow J(\bar{u})$.

Let us show that $\bar{u}$ is a solution of (NP). First we select an element $u \in H^{1 / 2}(\Gamma)$ such that $\alpha \leq u \leq \beta$, and we prove that $J(\bar{u}) \leq J(u)$. Indeed, it is clear that $u \circ g_{h}$ is a feasible control for $\left(\mathrm{NP}_{h}\right)$; consequently, $J_{h}\left(\bar{u}_{h}\right) \leq J_{h}\left(u \circ g_{h}\right)$. Furthermore, if we denote by $y_{h}$ the state associated to $u \circ g_{h}$, then (5.2) implies that

$$
\left\|y_{u}-y_{h}\right\|_{H^{3 / 2}\left(\Omega_{h}\right)} \leq C h\left[1+\|u\|_{H^{1 / 2}(\Gamma)}\right] .
$$

This along with (4.3) implies the convergence $J_{h}\left(u \circ g_{h}\right) \rightarrow J(u)$. Thus, we have

$$
J(\bar{u})=\lim _{h \rightarrow 0} J_{h}\left(\bar{u}_{h}\right) \leq \lim _{h \rightarrow 0} J_{h}\left(u \circ g_{h}\right)=J(u) .
$$

Finally, let us take $u \in L^{2}(\Gamma)$, with $\alpha \leq u \leq \beta$. There exists a sequence $\left\{u_{k}\right\}_{k=1}^{\infty} \subset$ $H^{1 / 2}(\Gamma)$ such that $u_{k} \rightarrow u$ in $L^{2}(\Gamma)$. Setting $\hat{u}_{k}=\operatorname{Proj}_{[\alpha, \beta]}\left(u_{k}\right)$, we have that $\left\{\hat{u}_{k}\right\}_{k=1}^{\infty}$ is still strongly convergent to $u$ in $L^{2}(\Gamma)$ and $\hat{u}_{k} \in H^{1 / 2}(\Gamma)$ is a feasible control for (NP) for every $k$; then $J(\bar{u}) \leq J\left(\hat{u}_{k}\right)$ for every $k$. Now, passing to the limit, we obtain $J(\bar{u}) \leq J(u)$. Since $u$ is an arbitrary feasible control of (NP), this completes the proof.

Now we consider the approximation of local minima of (NP) by local minima of problems $\left(\mathrm{NP}_{h}\right)$. First let us say that whenever we speak about a local minimum it must be understood as a local minimum in the sense of $L^{2}$; more precisely, it is the minimum among all feasible controls in an $L^{2}$-ball centered at the specific solution.

THEOREM 5.5. Let $\bar{u}$ be a strict local minimum of (NP); then there exists a family $\left\{\bar{u}_{h}\right\}$ such that each $\bar{u}_{h}$ is a local minimum of $\left(\mathrm{NP}_{h}\right)$ and $\bar{u}_{h} \circ g_{h}^{-1} \rightarrow \bar{u}$ weakly in $H^{1}(\Gamma)$. 
Proof. Let $\varepsilon>0$ be such that $\bar{u}$ is the only global solution of problem

$$
\left(\mathrm{NP}_{\varepsilon}\right)\left\{\begin{array}{l}
\min J(u)=\int_{\Omega} L\left(x, y_{u}(x)\right) d x+\frac{N}{2} \int_{\Gamma} u^{2}(x) d \sigma(x) \\
\text { subject to }\left(y_{u}, u\right) \in\left(L^{\infty}(\Omega) \cap H^{1}(\Omega)\right) \times L^{2}(\Gamma), \\
\alpha \leq u(x) \leq \beta \text { for a.e. } x \in \Gamma \text { and }\|u-\bar{u}\|_{L^{2}(\Gamma)} \leq \varepsilon .
\end{array}\right.
$$

Now for every $h \leq h_{0}$ we consider the problems

$$
\left(\mathrm{NP}_{h \varepsilon}\right)\left\{\begin{array}{l}
\min J_{h}(u)=\int_{\Omega_{h}} L\left(x, y_{h, u}(x)\right) d x+\frac{N}{2} \int_{\Gamma_{h}} u^{2}(x) d \sigma_{h}(x) \\
\text { subject to }\left(y_{h, u}, u\right) \in\left(L^{\infty}\left(\Omega_{h}\right) \cap H^{1}\left(\Omega_{h}\right)\right) \times L^{2}\left(\Gamma_{h}\right), \\
\alpha \leq u(x) \leq \beta \text { for a.e. } x \in \Gamma_{h} \text { and }\left\|u \circ g_{h}^{-1}-\bar{u}\right\|_{L^{2}(\Gamma)} \leq \varepsilon .
\end{array}\right.
$$

It is obvious that $\bar{u} \circ g_{h}$ is a feasible control for every problem $\left(\mathrm{NP}_{h \varepsilon}\right)$; therefore, there exists at least one solution $u_{h \varepsilon}$ of $\left(\mathrm{NP}_{h \varepsilon}\right)$. Let us prove that $u_{h \varepsilon} \circ g_{h}^{-1} \rightarrow \bar{u}$ weakly in $H^{1}(\Gamma)$ with $h \rightarrow 0$.

Since $\left\{u_{h \varepsilon} \circ g_{h}^{-1}\right\}_{0<h \leq h_{0}}$ is bounded in $L^{2}(\Gamma)$, we can take a subsequence, denoted in the same manner, and an element $\tilde{u} \in L^{2}(\Gamma)$ such that $u_{h \varepsilon} \circ g_{h}^{-1} \rightarrow \tilde{u}$ weakly in $L^{2}(\Gamma)$ with $h \rightarrow 0$. Let us denote by $y_{h \varepsilon} \in H^{3 / 2}\left(\Omega_{h}\right)$ the state associated to $u_{h \varepsilon}$ and consider an extension of $y_{h \varepsilon}$ to $\Omega$, still denoted by $y_{h \varepsilon}$, such that

$$
\left\|y_{h \varepsilon}\right\|_{H^{3 / 2}(\Omega)} \leq C\left\|y_{h \varepsilon}\right\|_{H^{3 / 2}\left(\Omega_{h}\right)} \forall h .
$$

The boundedness of $\left\{u_{h \varepsilon} \circ g_{h}^{-1}\right\}_{0<h \leq h_{0}}$ in $L^{2}(\Gamma)$ implies that $\left\{y_{h \varepsilon}\right\}$ is bounded in $H^{3 / 2}(\Omega)$. Therefore, by taking a subsequence, we can assume that

$$
y_{h \varepsilon} \rightarrow \tilde{y} \text { in } H^{3 / 2}(\Omega) \text { and } u_{h \varepsilon} \circ g_{h}^{-1} \rightarrow \tilde{u} \text { in } L^{2}(\Gamma) .
$$

Using the compactness of the embeddings $H^{3 / 2}(\Omega) \subset H^{1}(\Omega)$ and $H^{3 / 2}(\Omega) \subset L^{\infty}(\Omega)$, it is easy to prove that $\tilde{y}$ is the solution of (2.1) associated to the control $\tilde{u}$. On the other hand, each $u_{h \varepsilon} \circ g_{h}^{-1}$ is a feasible control for $\left(\mathrm{NP}_{\varepsilon}\right)$, and the set of feasible controls for this problem is convex and closed in $L^{2}(\Gamma)$; consequently, $\tilde{u}$ is also a feasible control for $\left(\mathrm{NP}_{\varepsilon}\right)$. From (5.2), the strong convergence $y_{h \varepsilon} \rightarrow \tilde{y}$ in $L^{\infty}(\Omega)$, the weak convergence $u_{h \varepsilon} \circ g_{h}^{-1} \rightarrow \tilde{u},(4.3)$, and the fact that $u_{h \varepsilon}$ is a solution of $\left(\mathrm{NP}_{h \varepsilon}\right)$ and $\bar{u} \circ g_{h}^{-1}$ is feasible for $\left(\mathrm{NP}_{\varepsilon}\right)$, we get

$$
J(\tilde{u}) \leq \liminf _{h \rightarrow 0} J_{h}\left(u_{h \varepsilon}\right) \leq \liminf _{h \rightarrow 0} J_{h}\left(\bar{u} \circ g_{h}^{-1}\right) \leq \limsup _{h \rightarrow 0} J_{h}\left(\bar{u} \circ g_{h}^{-1}\right)=J(\bar{u}) .
$$

The fact that $\bar{u}$ is the unique solution of $\left(\mathrm{NP}_{\varepsilon}\right)$ and the above inequalities lead to $\tilde{u}=\bar{u}$ and $J_{h}\left(u_{h \varepsilon}\right) \rightarrow J(\bar{u})$, which implies

$$
\lim _{h \rightarrow 0} \int_{\Gamma_{h}} u_{h \varepsilon}^{2}(x) d \sigma_{h}(x)=\int_{\Gamma} \bar{u}^{2}(x) d \sigma(x) .
$$

Using (4.3) once again, we have

$$
\lim _{h \rightarrow 0} \int_{\Gamma}\left(u_{h \varepsilon} \circ g_{h}^{-1}\right)^{2}(x) d \sigma(x)=\int_{\Gamma} \bar{u}^{2}(x) d \sigma(x) .
$$

This identity and the weak convergence imply the strong convergence $u_{h \varepsilon} \circ g_{h}^{-1} \rightarrow$ $\bar{u}$ in $L^{2}(\Gamma)$. A first consequence of this strong convergence is that the constraint 
$\left\|u \circ g_{h}^{-1}-\bar{u}\right\|_{L^{2}(\Gamma)} \leq \varepsilon$ is not active for the elements $u_{h \varepsilon}$ if $h$ is small enough. Therefore, $u_{h \varepsilon}$ is a local minimum of problem $\left(\mathrm{NP}_{h}\right)$ for $h$ small enough. Since $\left\{\left\|u_{h \varepsilon}\right\|_{L^{2}\left(\Gamma_{h}\right)}\right\}$ is bounded, we can use (4.13) and conclude that $\left\{u_{h \varepsilon} \circ g_{h}^{-1}\right\}$ is bounded in $H^{1}(\Gamma)$, and hence $u_{h \varepsilon} \circ g_{h}^{-1} \rightarrow \bar{u}$ weakly in $H^{1}(\Gamma)$ with $h \rightarrow 0$.

6. Error estimates for problems $\left(\mathbf{N P}_{\boldsymbol{h}}\right)$. In this section we assume that $\bar{u}_{h}$ is a local minimum of $\left(\mathrm{NP}_{h}\right)$ for every $h \leq h_{0}$, such that $\bar{u}_{h} \circ g_{h}^{-1}$ converges weakly in $H^{1}(\Gamma)$ to a local minimum $\bar{u}$ of (NP) with $h \rightarrow 0$; see Theorems 5.4 and 5.5. The goal of this section is to derive estimates of $\left\|\bar{u}-\bar{u}_{h} \circ g_{h}^{-1}\right\|_{L^{2}(\Gamma)}$, which are established in the following theorem.

TheOrEm 6.1. Let $\bar{u}$ and $\bar{u}_{h}$ be as above, and let us denote by $\bar{y}, \bar{y}_{h}$ and $\bar{\varphi}, \bar{\varphi}_{h}$ the states and adjoint states associated to $\bar{u}$ and $\bar{u}_{h}$, respectively. Let us assume that the second order sufficient optimality condition (3.6) is fulfilled. Then there exists a constant $C$ independent of $h$ such that the following estimates hold:

$$
\begin{aligned}
& \left\|\bar{u}-\bar{u}_{h} \circ g_{h}^{-1}\right\|_{L^{2}(\Gamma)} \leq C h^{5 / 3}, \\
& \left\|\bar{y}-\bar{y}_{h}\right\|_{H^{s}\left(\Omega_{h}\right)}+\left\|\bar{\varphi}-\bar{\varphi}_{h}\right\|_{H^{s}\left(\Omega_{h}\right)} \leq C h^{\min \{5,6-2 s\} / 3} \quad \forall 0 \leq s \leq \frac{3}{2} .
\end{aligned}
$$

Proof. By taking $v=\bar{u}_{h} \circ g_{h}^{-1}$ in (3.3) and $v_{h}=\bar{u} \circ g_{h}$ in (4.9), we get

$$
J^{\prime}(\bar{u})\left(\bar{u}_{h} \circ g_{h}^{-1}-\bar{u}\right)=\int_{\Gamma}(\bar{\varphi}+N \bar{u})\left(\bar{u}_{h} \circ g_{h}^{-1}-\bar{u}\right) d \sigma \geq 0
$$

and

$$
J_{h}^{\prime}\left(\bar{u}_{h}\right)\left(\bar{u} \circ g_{h}-\bar{u}_{h}\right)=\int_{\Gamma_{h}}\left(\bar{\varphi}_{h}+N \bar{u}_{h}\right)\left(\bar{u} \circ g_{h}-\bar{u}_{h}\right) d \sigma_{h} \geq 0 .
$$

We rewrite inequality (6.4) as follows:

$$
J^{\prime}\left(\bar{u}_{h} \circ g_{h}^{-1}\right)\left(\bar{u}-\bar{u}_{h} \circ g_{h}^{-1}\right)+\left[J_{h}^{\prime}\left(\bar{u}_{h}\right)\left(\bar{u} \circ g_{h}-\bar{u}_{h}\right)-J^{\prime}\left(\bar{u}_{h} \circ g_{h}^{-1}\right)\left(\bar{u}-\bar{u}_{h} \circ g_{h}^{-1}\right)\right] \geq 0 .
$$

From (6.3) and (6.5) we obtain

$$
\left[J^{\prime}\left(\bar{u}_{h} \circ g_{n}^{-1}\right)-J^{\prime}(\bar{u})\right]\left(\bar{u}_{h} \circ g_{h}^{-1}-\bar{u}\right) \leq J_{h}^{\prime}\left(\bar{u}_{h}\right)\left(\bar{u} \circ g_{h}-\bar{u}_{h}\right)-J^{\prime}\left(\bar{u}_{h} \circ g_{h}^{-1}\right)\left(\bar{u}-\bar{u}_{h} \circ g_{h}^{-1}\right) .
$$

By applying the mean value theorem we obtain the existence of an element $v_{h}=$ $\bar{u}+\theta_{h}\left(\bar{u}_{h} \circ g_{n}^{-1}-\bar{u}\right)$ such that

$$
J^{\prime \prime}\left(v_{h}\right)\left(\bar{u}_{h} \circ g_{h}^{-1}-\bar{u}\right)^{2} \leq J_{h}^{\prime}\left(\bar{u}_{h}\right)\left(\bar{u} \circ g_{h}-\bar{u}_{h}\right)-J^{\prime}\left(\bar{u}_{h} \circ g_{h}^{-1}\right)\left(\bar{u}-\bar{u}_{h} \circ g_{h}^{-1}\right) .
$$

This inequality plays the central role in the derivation of (6.1). The proof is divided into two parts. First we use the second order optimality condition (3.6), or, more precisely, its equivalent formulation (3.8), to estimate the left-hand side of (6.6) from below. In the second part we estimate the right-hand side in terms of $h$ from above. The inequality (6.2) is an immediate consequence of (6.1) combined with the estimates (5.2) and (5.24).

Lower bounds for (6.6). Let us prove that $\bar{u}_{h} \circ g_{h}^{-1}-\bar{u} \in C_{\bar{u}}^{\vartheta}$ for every $h$ small enough. Indeed, $\bar{u}_{h} \circ g_{h}^{-1}-\bar{u}$ obviously satisfies conditions (3.5). Let us check that $\left(\bar{u}_{h} \circ g_{h}^{-1}-\bar{u}\right)(x)=0$ at the points $x$ where $|\bar{\varphi}(x)+N \bar{u}(x)|>\vartheta$. First, we observe 
that the weak convergence $\bar{u}_{h} \circ g_{h}^{-1} \rightarrow \bar{u}$ in $H^{1}(\Gamma)$ implies the strong convergence in $C(\Gamma)$. On the other hand, from (5.24) with $s=3 / 2$ we get

$$
\begin{aligned}
& \left\|\bar{\varphi}-\bar{\varphi}_{h}\right\|_{C\left(\bar{\Omega}_{h}\right)} \leq C_{1}\left\|\bar{\varphi}-\bar{\varphi}_{h}\right\|_{H^{3 / 2}\left(\Omega_{h}\right)} \\
\leq & C_{2}\left\{\left\|\bar{u}-\bar{u}_{h} \circ g_{h}^{-1}\right\|_{L^{2}(\Gamma)}+h\left[\|\bar{u}\|_{H^{1 / 2}(\Gamma)}+1\right]\right\} \rightarrow 0 \text { with } h \rightarrow 0 .
\end{aligned}
$$

This inequality implies the strong convergence $\bar{\varphi}_{h} \circ g_{h}^{-1} \rightarrow \bar{\varphi}$ in $C(\Gamma)$. Therefore, there exists $h_{1}>0$ such that

$$
\left\|\left(\bar{\varphi}_{h} \circ g_{h}^{-1}+\bar{u}_{h} \circ g_{h}^{-1}\right)-(\bar{\varphi}+N \bar{u})\right\|_{C(\Gamma)}<\frac{\vartheta}{2} \quad \forall h \leq h_{1} .
$$

Thus, if $(\bar{\varphi}+N \bar{u})(x)>\vartheta$, then $\left(\bar{\varphi}_{h} \circ g_{h}^{-1}+\bar{u}_{h} \circ g_{h}^{-1}\right)(x)>\vartheta / 2$ for every $h \leq h_{1}$. Using the identities (3.4) and (4.11), we have that $\bar{u}(x)=\bar{u}_{h} \circ g_{h}^{-1}(x)=\alpha$; therefore, $\left(\bar{u}(x)-\bar{u}_{h} \circ g_{h}^{-1}\right)(x)=0$. Analogously we can prove that $(\bar{\varphi}+N \bar{u})(x)<-\vartheta$ leads to $\bar{u}(x)=\bar{u}_{h} \circ g_{h}^{-1}(x)=\beta$ and then $\left(\bar{u}(x)-\bar{u}_{h} \circ g_{h}^{-1}\right)(x)=0$ as well. This proves that $\bar{u}_{h} \circ g_{h}^{-1}-\bar{u} \in C_{\bar{u}}^{\vartheta}$, and hence (3.8) implies

$$
J^{\prime \prime}(\bar{u})\left(\bar{u}_{h} \circ g_{h}^{-1}-\bar{u}\right)^{2} \geq \mu\left\|\bar{u}_{h} \circ g_{h}^{-1}-\bar{u}\right\|_{L^{2}(\Gamma)}^{2} \quad \forall h \leq h_{2} .
$$

For the elements $v_{h}$ in (6.6) we have that $v_{h} \rightarrow \bar{u}$ in $C(\Gamma)$ with $h \rightarrow 0$. On the other hand, the mapping $J$ is of class $C^{2}$ in $L^{2}(\Gamma)$; therefore, there exists $0<h_{2} \leq h_{1}$ such that

$$
\left|\left[J^{\prime \prime}(\bar{u})-J^{\prime \prime}\left(v_{h}\right)\right]\left(\bar{u}_{h} \circ g_{h}^{-1}-\bar{u}\right)^{2}\right| \leq \frac{\mu}{2}\left\|\bar{u}_{h} \circ g_{h}^{-1}-\bar{u}\right\|_{L^{2}(\Gamma)}^{2} \quad \forall h \leq h_{2} .
$$

This inequality combined with (6.9) leads to

$$
J^{\prime \prime}\left(v_{h}\right)\left(\bar{u}_{h} \circ g_{h}^{-1}-\bar{u}\right)^{2} \geq \frac{\mu}{2}\left\|\bar{u}_{h} \circ g_{h}^{-1}-\bar{u}\right\|_{L^{2}(\Gamma)}^{2} .
$$

Upper bounds for (6.6). Let us define $y, \varphi \in H^{2}(\Omega)$ as the solutions of the equations

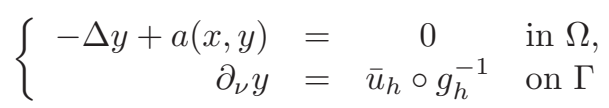

and

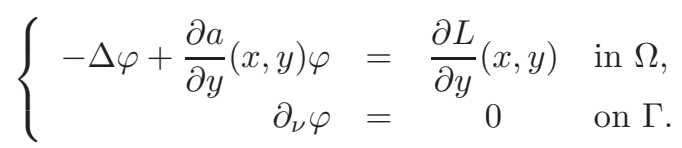

Then we have

$$
J^{\prime}\left(\bar{u}_{h} \circ g_{h}^{-1}\right)\left(\bar{u}-\bar{u}_{h} \circ g_{h}^{-1}\right)=\int_{\Gamma}\left(\varphi+N \bar{u}_{h} \circ g_{h}^{-1}\right)\left(\bar{u}-\bar{u}_{h} \circ g_{h}^{-1}\right) d \sigma .
$$

Copyright $@$ by SIAM. Unauthorized reproduction of this article is prohibited. 
From (6.3) and (6.13) and taking into account (4.2), (4.3), and (5.9), we get

$$
\begin{aligned}
& \left|J_{h}^{\prime}\left(\bar{u}_{h}\right)\left(\bar{u} \circ g_{h}-\bar{u}_{h}\right)-J^{\prime}\left(\bar{u}_{h} \circ g_{h}^{-1}\right)\left(\bar{u}-\bar{u}_{h} \circ g_{h}^{-1}\right)\right| \\
= & \left|\int_{\Gamma_{h}}\left(\bar{\varphi}_{h}+N \bar{u}_{h}\right)\left(\bar{u} \circ g_{h}-\bar{u}_{h}\right) d \sigma_{h}-\int_{\Gamma}\left(\varphi+N \bar{u}_{h} \circ g_{h}^{-1}\right)\left(\bar{u}-\bar{u}_{h} \circ g_{h}^{-1}\right) d \sigma\right| \\
\leq & \int_{\Gamma_{h}}\left|\left(\bar{\varphi}_{h}+N \bar{u}_{h}\right)-\left(\varphi \circ g_{h}+N \bar{u}_{h}\right)\right|\left|\bar{u} \circ g_{h}-\bar{u}_{h}\right| d \sigma_{h} \\
& +C h^{2} \int_{\Gamma}\left|\varphi+N \bar{u}_{h} \circ g_{h}^{-1} \| \bar{u}-\bar{u}_{h} \circ g_{h}^{-1}\right| d \sigma \\
\leq & \left\{\left\|\bar{\varphi}_{h}-\varphi\right\|_{L^{2}\left(\Gamma_{h}\right)}+\left\|\varphi \circ g_{h}-\varphi\right\|_{L^{2}\left(\Gamma_{h}\right)}\right\}\left\|\bar{u} \circ g_{h}-\bar{u}_{h}\right\|_{L^{2}\left(\Gamma_{h}\right)} \\
& +C h^{2}\left\|\varphi+N \bar{u}_{h} \circ g_{h}^{-1}\right\|_{L^{2}(\Gamma)}\left\|\bar{u}-\bar{u}_{h} \circ g_{h}^{-1}\right\|_{L^{2}(\Gamma)} \\
(6.14) \leq & C\left(h^{5 / 3}+h^{2}\right)\left\|\bar{u}-\bar{u}_{h} \circ g_{h}^{-1}\right\|_{L^{2}(\Gamma)} \leq C h^{5 / 3}\left\|\bar{u}-\bar{u}_{h} \circ g_{h}^{-1}\right\|_{L^{2}(\Gamma)},
\end{aligned}
$$

the last estimate being a consequence of (5.35).

Finally, (6.6), (6.10), and (6.14) lead to (6.1), which completes the proof.

7. Dirichlet control problem. Now we introduce the Dirichlet control problem

$$
(\mathrm{DP})\left\{\begin{array}{l}
\min J(u)=\int_{\Omega} L\left(x, y_{u}(x)\right) d x+\frac{N}{2} \int_{\Gamma} u^{2}(x) d \sigma(x) \\
\text { subject to }\left(y_{u}, u\right) \in\left(L^{\infty}(\Omega) \cap H^{1 / 2}(\Omega)\right) \times L^{\infty}(\Gamma), \\
\alpha \leq u(x) \leq \beta \text { for a.e. } x \in \Gamma,
\end{array}\right.
$$

where the state $y_{u}$ associated to the control $u$ is the solution of the Dirichlet problem

$$
\left\{\begin{array}{rlll}
-\Delta y+a(x, y) & =0 & & \text { in } \Omega \\
y & = & & \text { on } \Gamma .
\end{array}\right.
$$

The following hypotheses are assumed for this problem.

(D1) $\Omega$ is an open, convex, and bounded domain in $\mathbb{R}^{2}$, with the boundary $\Gamma$ of class $C^{2}$. Moreover, we assume that $N>0$ and $-\infty<\alpha<\beta<+\infty$.

(D2) We assume that $L$ and $a$ satisfy (2.2), and (2.3) is replaced by

$$
\frac{\partial a}{\partial y}(x, y) \geq 0 \text { for a.e. } x \in \Omega \text { and } \forall y \in \mathbb{R} .
$$

We say that an element $y_{u} \in L^{\infty}(\Omega)$ is a solution of (7.1) if the following integral identity is fulfilled:

$$
\int_{\Omega}-y \Delta w d x+\int_{\Omega} a(x, y) w d x=\int_{\Gamma} u \partial_{\nu} w d \sigma \quad \forall w \in H^{2}(\Omega) \cap H_{0}^{1}(\Omega),
$$

where $\partial_{\nu}$ denotes the normal derivative on the boundary $\Gamma$. This is the classical definition of a weak solution by transposition. The following result proved by Casas and Raymond [5] is valid for any convex domain $\Omega$. If the domain is not convex, then some smoothness of $\Gamma$ is required; $\Gamma$ of class $C^{1,1}$ is enough.

TheOREM 7.1. For every $u \in L^{\infty}(\Gamma)$ the state equation (7.1) has a unique solution $y_{u} \in L^{\infty}(\Omega) \cap H^{1 / 2}(\Omega)$. Moreover, the following Lipschitz properties hold:

$$
\begin{aligned}
& \left\|y_{u}-y_{v}\right\|_{L^{\infty}(\Omega)} \leq\|u-v\|_{L^{\infty}(\Gamma)}, \\
& \left\|y_{u}-y_{v}\right\|_{H^{1 / 2}(\Omega)} \leq C\|u-v\|_{L^{2}(\Gamma)} \quad \forall u, v \in L^{\infty}(\Gamma) .
\end{aligned}
$$

Copyright $@$ by SIAM. Unauthorized reproduction of this article is prohibited. 
Finally, if $u_{n} \rightarrow u$ weakly ${ }^{\star}$ in $L^{\infty}(\Gamma)$, then $y_{u_{n}} \rightarrow y_{u}$ strongly in $L^{r}(\Omega)$ for all $r<+\infty$.

Under the assumptions (D1) and (D2), it can be shown by standard arguments that problem (DP) has at least one solution. Since (DP) is not convex, we cannot expect any uniqueness of solutions. Moreover, (DP) may have some local solutions. We formulate the optimality conditions satisfied by such local solutions. To this end, we analyze the differentiability of the cost functional $J$.

Under the assumption (D2), $J: L^{\infty}(\Gamma) \longrightarrow \mathbb{R}$ is of class $C^{2}$, and

$$
J^{\prime}(u) v=\int_{\Gamma}\left(N u-\partial_{\nu} \varphi_{u}\right) v d \sigma
$$

where $y_{u}$ is the state associated to $u$ and $\varphi_{u} \in H^{2}(\Omega)$ is the unique solution of the problem

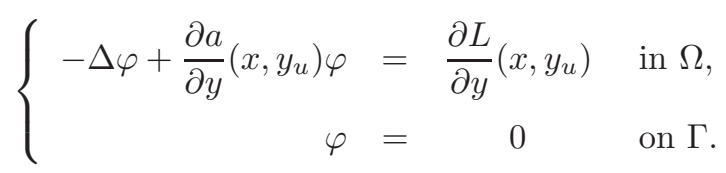

Furthermore, we have

$$
J^{\prime \prime}(u)\left(v_{1}, v_{2}\right)=\int_{\Omega}\left[\frac{\partial^{2} L}{\partial y^{2}}\left(x, y_{u}\right) z_{v_{1}} z_{v_{2}}-\varphi_{u} \frac{\partial^{2} a}{\partial y^{2}}\left(x, y_{u}\right) z_{v_{1}} z_{v_{2}}\right] d x+\int_{\Gamma} N v_{1} v_{2} d \sigma
$$

where $z_{v_{i}}, i=1,2$, satisfy

$$
\left\{\begin{array}{rlll}
-\Delta z_{v_{i}}+\frac{\partial a}{\partial y}\left(x, y_{u}\right) z_{v_{i}} & = & 0 & \text { in } \Omega \\
z_{v_{i}} & = & v_{i} & \text { on } \Gamma .
\end{array}\right.
$$

Using (7.5), we obtain the necessary optimality conditions for (DP).

TheOrem 7.2. Let $\bar{u}$ be a local minimum of (DP). Then $\bar{u} \in W^{1-1 / p, p}(\Gamma)$ for every $1 \leq p<+\infty$, and there exist elements $\bar{y} \in W^{1, p}(\Omega)$ and $\bar{\varphi} \in W^{2, p}(\Omega)$ such that

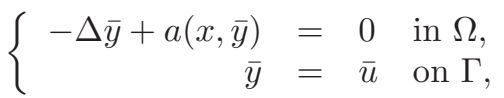

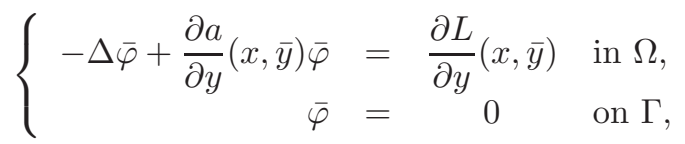

$$
\begin{aligned}
& \int_{\Gamma}\left(N \bar{u}(x)-\partial_{\nu} \bar{\varphi}(x)\right)(v(x)-\bar{u}(x)) d \sigma(x) \geq 0 \quad \forall \alpha \leq v \leq \beta .
\end{aligned}
$$

The proof of the theorem is given in [5].

In order to establish the second order optimality conditions, we define the cone of critical directions

$$
\begin{gathered}
C_{\bar{u}}=\left\{v \in L^{2}(\Gamma) \text { satisfying }(7.12) \text { and } v(x)=0 \text { if }\left|N \bar{u}(x)-\partial_{\nu} \bar{\varphi}(x)\right|>0\right\}, \\
v v(x)=\left\{\begin{array}{ll}
\geq 0 & \text { if } \bar{u}(x)=\alpha, \\
\leq 0 & \text { if } \bar{u}(x)=\beta
\end{array} \quad \text { for a.a. } x \in \Gamma .\right.
\end{gathered}
$$

Now we formulate the second order necessary and sufficient optimality conditions.

Copyright $@$ by SIAM. Unauthorized reproduction of this article is prohibited. 
THEOREM 7.3. If $\bar{u}$ is a local solution of (DP), then $J^{\prime \prime}(\bar{u}) v^{2} \geq 0$ holds for all $v \in C_{\bar{u}}$. Conversely, if $\bar{u}$ is an admissible control for problem (DP) satisfying the first order optimality conditions given in Theorem 7.2 and the coercivity condition

$$
J^{\prime \prime}(\bar{u}) v^{2}>0 \quad \forall v \in C_{\bar{u}} \backslash\{0\},
$$

then there exist $\delta>0$ and $\rho>0$ such that

$$
J(u) \geq J(\bar{u})+\frac{\delta}{2}\|u-\bar{u}\|_{L^{2}(\Gamma)}^{2}
$$

for all $u$ such that $\alpha \leq u \leq \beta$ and $\|u-\bar{u}\|_{L^{2}(\Gamma)} \leq \rho$.

The inequality (7.14) is strong when compared with the corresponding inequality of [5]. Indeed, here we claim that (7.13) implies that $\bar{u}$ is a strict local minimum of (DP) in the sense of the $L^{2}(\Gamma)$-topology. In [5] it is shown that condition (7.13) leads to the strict local optimality of $\bar{u}$ in the sense of the $L^{\infty}(\Gamma)$-topology. A more general result is proved in [2] for a distributed control problem, but in such a case once again only the local optimality in the sense of the $L^{\infty}(\Omega)$-topology is shown. Here we can improve the result because the control appears in a quadratic form within the cost functional.

Proof. The necessary condition is easy to obtain. Let us prove that (7.13) implies (7.14). We proceed by contradiction. Let us assume that there is no pair $(\delta, \rho)$, with $\rho, \delta>0$, such that (7.14) holds. Then for every integer $k$, there exists a feasible control of (DP), $u_{k} \in L^{2}(\Gamma)$, such that

$$
\left\|u_{k}-\bar{u}\right\|_{L^{2}(\Gamma)}<\frac{1}{k} \text { and } J\left(u_{k}\right)<J(\bar{u})+\frac{1}{k}\left\|u_{k}-\bar{u}\right\|_{L^{2}(\Gamma)}^{2} .
$$

Let us define

$$
\lambda_{k}=\left\|u_{k}-\bar{u}\right\|_{L^{2}(\Gamma)} \text { and } v_{k}=\frac{1}{\lambda_{k}}\left(u_{k}-\bar{u}\right) ; \text { hence }\left\|v_{k}\right\|_{L^{2}(\Gamma)}=1 .
$$

By taking a subsequence, if necessary, there exists $v \in L^{2}(\Gamma)$ such that $v_{k} \rightarrow v$ weakly in $L^{2}(\Gamma)$. The proof is divided into three steps: first we prove that $v \in C_{\bar{u}}$, then we deduce that $v=0$, and finally we get the contradiction.

Step 1. $v \in C_{\bar{u}}$. Since $\alpha \leq u_{k} \leq \beta$, it is obvious that every $v_{k}$ satisfies (7.12). Also we have that the set of functions of $L^{2}(\Gamma)$ satisfying (7.12) is convex and closed; therefore, $v$ satisfies (7.12) as well. This implies

$$
\left(N \bar{u}(x)-\partial_{\nu} \bar{\varphi}(x)\right) v(x)=\left|N \bar{u}(x)-\partial_{\nu} \bar{\varphi}(x)\right||v(x)| \quad \text { a.e. on } \Gamma .
$$

Indeed, it is well known that (7.11) implies that $N \bar{u}(x)-\partial_{\nu} \bar{\varphi}(x) \geq 0$ if $\bar{u}(x)=\alpha$ and $N \bar{u}(x)-\partial_{\nu} \bar{\varphi}(x) \leq 0$ if $\bar{u}(x)=\beta$. This property and (7.12) lead to (7.17).

On the other hand, from (7.15) we get

$$
\frac{1}{k}\left\|u_{k}-\bar{u}\right\|_{L^{2}(\Gamma)}^{2}>J\left(u_{k}\right)-J(\bar{u})=J\left(\bar{u}+\lambda_{k} v_{k}\right)-J(\bar{u})=J^{\prime}\left(\bar{u}+\theta_{k} \lambda_{k} v_{k}\right) v_{k}
$$

for some $0<\theta_{k}<1$. From (7.5) we have that

$$
J^{\prime}\left(\bar{u}+\theta_{k} \lambda_{k} v_{k}\right) v_{k}=\int_{\Gamma}\left(N\left[\bar{u}+\theta_{k} \lambda_{k} v_{k}\right]-\partial_{\nu} \varphi_{k}\right) v_{k} d \sigma,
$$

Copyright (C) by SIAM. Unauthorized reproduction of this article is prohibited. 
where $\varphi_{k}$ is the solution of (7.6) with $y_{u}$ replaced by $y_{k}, y_{k}$ being the state associated to $\bar{u}+\theta_{k} \lambda_{k} v_{k}=\bar{u}+\theta_{k}\left(u_{k}-\bar{u}\right)$. Since $\alpha \leq \bar{u}+\theta_{k}\left(u_{k}-\bar{u}\right) \leq \beta$ and $\bar{u}+\theta_{k}\left(u_{k}-\bar{u}\right) \rightarrow \bar{u}$ in $L^{2}(\Gamma)$ for $k \rightarrow+\infty$, we deduce, in view of (7.4), that $\left\{y_{k}\right\}_{k=1}^{\infty}$ is bounded in $L^{\infty}(\Gamma)$ and $y_{k} \rightarrow \bar{y}$ in $H^{1 / 2}(\Omega)$. Therefore, the sequence of adjoint states $\left\{\varphi_{k}\right\}_{k=1}^{\infty}$ converges to $\bar{\varphi}$ in $H^{2}(\Omega)$. Hence, we can pass to the limit in (7.19) and use (7.18) to deduce that

$$
\int_{\Gamma}\left(N \bar{u}-\partial_{\nu} \bar{\varphi}\right) v d \sigma \leq 0 .
$$

This identity and (7.17) imply that $v(x)=0$ if $\left|N \bar{u}(x)-\partial_{\nu} \bar{\varphi}(x)\right|>0$. Thus, we have that $v \in C_{\bar{u}}$.

Step 2. $v=0$. Using again (7.15), we obtain

$$
\begin{aligned}
& \frac{\lambda_{k}^{2}}{k}=\frac{1}{k}\left\|u_{k}-\bar{u}\right\|_{L^{2}(\Gamma)}^{2}>J\left(u_{k}\right)-J(\bar{u})=J\left(\bar{u}+\lambda_{k} v_{k}\right)-J(\bar{u}) \\
& =\lambda_{k} J^{\prime}(\bar{u}) v_{k}+\frac{\lambda_{k}^{2}}{2} J^{\prime \prime}\left(\bar{u}+\theta_{k} \lambda_{k} v_{k}\right) v_{k}^{2} \geq \frac{\lambda_{k}^{2}}{2} J^{\prime \prime}\left(\bar{u}+\theta_{k} \lambda_{k} v_{k}\right) v_{k}^{2},
\end{aligned}
$$

the last inequality being a consequence of (7.11); indeed,

$$
\lambda_{k} J^{\prime}(\bar{u}) v_{k}=J^{\prime}(\bar{u})\left(u_{k}-\bar{u}\right)=\int_{\Gamma}\left(N \bar{u}-\partial_{\nu} \bar{\varphi}\right)\left(u_{k}-\bar{u}\right) d \sigma \geq 0 .
$$

Inequality (7.20) implies that

$$
\frac{2}{k}>J^{\prime \prime}\left(\bar{u}+\theta_{k} \lambda_{k} v_{k}\right) v_{k}^{2} .
$$

Once again, we denote by $y_{k}$ and $\varphi_{k}$ the state and adjoint state evaluated for $\bar{u}+$ $\theta_{k} \lambda_{k} v_{k}=\bar{u}+\theta_{k}\left(u_{k}-\bar{u}\right)$. Also, we define $z_{k}$ and $z_{v}$ as the elements of $H^{1 / 2}(\Omega)$ satisfying

$$
\left\{\begin{array}{rll}
-\Delta z_{k}+\frac{\partial a}{\partial y}\left(x, y_{k}\right) z_{k} & =0 & \text { in } \Omega \\
z_{k} & =v_{k} & \text { on } \Gamma
\end{array}\right.
$$

and

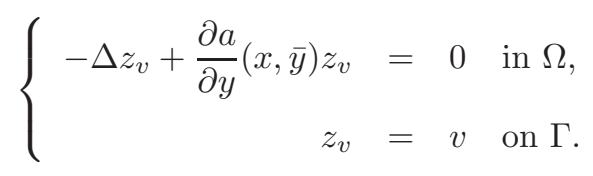

Then $z_{k} \rightarrow z_{v}$ weakly in $H^{1 / 2}(\Omega)$ and hence strongly in $L^{2}(\Omega)$. Moreover, $y_{k} \rightarrow \bar{y}$ in $H^{1 / 2}(\Omega)$ and $\varphi_{k} \rightarrow \bar{\varphi}$ in $H^{2}(\Omega)$. Now, recalling the expression of the second derivative of $J$ given in (7.7), we get

$$
J^{\prime \prime}\left(\bar{u}+\theta_{k} \lambda_{k} v_{k}\right) v_{k}^{2}=\int_{\Omega}\left[\frac{\partial^{2} L}{\partial y^{2}}\left(x, y_{k}\right) z_{k}^{2}-\varphi_{k} \frac{\partial^{2} a}{\partial y^{2}}\left(x, y_{k}\right) z_{k}^{2}\right] d x+N \int_{\Gamma} v_{k}^{2} d \sigma .
$$

Passing to the limit in this expression and using (7.21), we obtain

$$
\begin{aligned}
J^{\prime \prime}(\bar{u}) v^{2}=\int_{\Omega}\left[\frac{\partial^{2} L}{\partial y^{2}}(x, \bar{y}) z_{v}^{2}\right. & \left.-\bar{\varphi} \frac{\partial^{2} a}{\partial y^{2}}(x, \bar{y}) z_{v}^{2}\right] d x+N \int_{\Gamma} v^{2} d \sigma \\
& \leq \liminf _{k \rightarrow \infty} J^{\prime \prime}\left(\bar{u}+\theta_{k} \lambda_{k} v_{k}\right) v_{k}^{2} \leq 0 .
\end{aligned}
$$

Copyright $@$ by SIAM. Unauthorized reproduction of this article is prohibited. 
Since $v \in C_{\bar{u}}$, according to (7.13) this is possible only if $v=0$.

Step 3. Final contradiction. Using two facts, $v_{k} \rightarrow v=0$ and $\left\|v_{k}\right\|_{L^{2}(\Gamma)}=1$, we deduce from (7.21) and (7.24) the following contradiction:

$$
0<N \leq \liminf _{k \rightarrow \infty} J^{\prime \prime}\left(\bar{u}+\theta_{k} \lambda_{k} v_{k}\right) v_{k}^{2} \leq 0
$$

We conclude this section with the following result that provides an equivalent formulation of (7.13), which is more useful for our purposes.

THEOREM 7.4. Let $\bar{u}$ be a feasible control of problem (DP) satisfying the first order optimality conditions (7.9)-(7.11). Then the condition (7.13) holds if and only if

$$
\exists \mu>0 \text { and } \vartheta>0 \text { such that } J^{\prime \prime}(\bar{u}) v^{2} \geq \mu\|v\|_{L^{2}(\Gamma)}^{2} \forall v \in C_{\bar{u}}^{\vartheta}
$$

where

$$
C_{\bar{u}}^{\vartheta}=\left\{v \in L^{2}(\Gamma) \text { satisfying }(7.12) \text { and } v(x)=0 \text { if }\left|N \bar{u}(x)-\partial_{\nu} \bar{\varphi}(x)\right|>\vartheta\right\} .
$$

Proof. Since $C_{\bar{u}} \subset C_{\bar{u}}^{\vartheta}$ for any $\vartheta>0$, it is obvious that (7.26) implies (7.13). Let us prove the reciprocal implication. We proceed again by contradiction. We assume that $(7.13)$ holds, but there is no pair of positive numbers $(\mu, \vartheta)$ such that $(7.26)$ is fulfilled. Then for every integer $k$ there exists and element $v_{k} \in C_{\bar{u}}^{1 / k}$ such that

$$
J^{\prime \prime}(\bar{u}) v_{k}^{2}<\frac{1}{k}\left\|v_{k}\right\|_{L^{2}(\Gamma)}^{2} .
$$

Dividing $v_{k}$ by its norm and denoting the quotient by $v_{k}$ again, and taking a subsequence if necessary, we have that

$$
v_{k} \in C_{\bar{u}}^{1 / k}, \quad\left\|v_{k}\right\|_{L^{2}(\Gamma)}=1, \quad v_{k} \rightarrow v \text { in } L^{2}(\Gamma), \quad J^{\prime \prime}(\bar{u}) v_{k}^{2}<\frac{1}{k} .
$$

Arguing as in the proof of Theorem 7.3, we obtain that $v$ satisfies (7.12). On the other hand, from the fact that $v_{k} \in C_{\bar{u}}^{1 / k}$ and denoting by $\Gamma_{k}$ the subset of $\Gamma$ formed by those points $x$ such that $\left|N \bar{u}(x)-\partial_{\nu} \bar{\varphi}(x)\right| \leq 1 / k$, we get

$$
\begin{array}{r}
\int_{\Gamma}\left(N \bar{u}-\partial_{\nu} \bar{\varphi}\right) v d \sigma=\lim _{k \rightarrow \infty} \int_{\Gamma}\left(N \bar{u}-\partial_{\nu} \bar{\varphi}\right) v_{k} d \sigma \\
\leq \liminf _{k \rightarrow \infty} \int_{\Gamma}\left|N \bar{u}-\partial_{\nu} \bar{\varphi}\right|\left|v_{k}\right| d \sigma \leq \liminf _{k \rightarrow \infty} \frac{1}{k} \int_{\Gamma_{k}}\left|v_{k}\right| d \sigma=0 .
\end{array}
$$

This inequality and the fact that $v$ satisfies (7.12) imply that $v$ vanishes whenever $\left|N \bar{u}(x)-\partial_{\nu} \bar{\varphi}(x)\right|>0$; hence $v \in C_{\bar{u}}$. Now (7.13) implies that $J^{\prime \prime}(\bar{u})>0$ if $v \not \equiv 0$. But from (7.27) we deduce that

$$
J^{\prime \prime}(\bar{u}) v^{2} \leq \liminf _{k \rightarrow \infty} J^{\prime \prime}(\bar{u}) v_{k}^{2} \leq 0 .
$$

Consequently, we have that $v \equiv 0$. However, if we argue as in the proof of Theorem 7.3 , we have that $0<N \leq \liminf _{k \rightarrow \infty} J^{\prime \prime}(\bar{u}) v_{k}^{2} \leq 0$, which is a contradiction. 
8. Control problem $\left(\mathbf{D P}_{\boldsymbol{h}}\right)$. Now we consider $\Omega_{h}$ as defined in section 4 . In the domain $\Omega_{h}$ we define the problem $\left(\mathrm{DP}_{h}\right)$ as

$$
\left(\mathrm{DP}_{h}\right)\left\{\begin{array}{l}
\min J_{h}(u)=\int_{\Omega_{h}} L\left(x, y_{h, u}(x)\right) d x+\frac{N}{2} \int_{\Gamma_{h}} u^{2}(x) d \sigma_{h}(x) \\
\text { subject to }\left(y_{h, u}, u\right) \in\left(L^{\infty}\left(\Omega_{h}\right) \cap H^{1 / 2}\left(\Omega_{h}\right)\right) \times L^{\infty}\left(\Gamma_{h}\right), \\
\alpha \leq u(x) \leq \beta \quad \text { for a.e. } x \in \Gamma_{h},
\end{array}\right.
$$

where $y_{h, u}$ is the solution of the problem

$$
\left\{\begin{array}{rlll}
-\Delta y+a(x, y) & =0 & \text { in } \Omega_{h} \\
y & = & & \text { on } \Gamma_{h}
\end{array}\right.
$$

Theorem 7.1 can be applied to (8.1) to get the existence and uniqueness of a solution $y_{h, u} \in H^{1 / 2}\left(\Omega_{h}\right) \cap L^{\infty}\left(\Omega_{h}\right)$. Moreover, inequalities (7.4) hold. $\left(\mathrm{DP}_{h}\right)$ has at least one global solution and possibly there are some other local solutions of $\left(\mathrm{DP}_{h}\right)$. For each local solution we have the first order optimality conditions analogous to the conditions in Theorem 7.2.

THEOREM 8.1. Let $\bar{u}_{h}$ be a local minimum of $\left(\mathrm{DP}_{h}\right)$. Then $\bar{u}_{h} \in H^{1 / 2}\left(\Gamma_{h}\right)$ and there exist elements $\bar{y}_{h} \in H^{1}\left(\Omega_{h}\right)$ and $\bar{\varphi}_{h} \in H^{2}\left(\Omega_{h}\right)$ such that

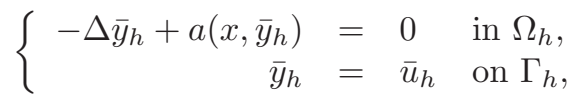

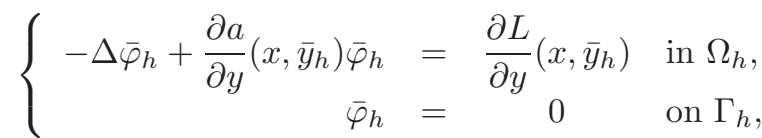

$$
\begin{aligned}
& \int_{\Gamma_{h}}\left(N \bar{u}_{h}(x)-\partial_{\nu_{h}} \bar{\varphi}_{h}(x)\right)\left(v_{h}(x)-\bar{u}_{h}(x)\right) d \sigma_{h}(x) \geq 0 \quad \forall \alpha \leq v_{h} \leq \beta .
\end{aligned}
$$

Remark 8.2. We observe that $\bar{u}_{h}$ is less regular than $\bar{u}$. The same is true for $\bar{y}_{h}$ and $\bar{\varphi}_{h}$ with respect to $\bar{y}$ and $\bar{\varphi}$. The reason for the loss of regularity is the lack of regularity of $\Gamma_{h}$. $\Gamma$ is of class $C^{2}$, and consequently we can deduce the $W^{2, p}(\Omega)$ regularity of $\bar{\varphi}$ for any $p<+\infty$ (see, for instance, Grisvard [7]), which leads to the $W^{1-1 / p}(\Gamma)$ regularity of $\bar{u}$ and consequently to the $W^{1, p}(\Omega)$ regularity of $\bar{y}$. Using the results for polygonal domains of [7], we can establish the $W^{2, p}(\Omega)$ regularity of $\bar{\varphi}_{h}$ for some $2<p$, with $p$ depending on the angles of $\Omega_{h}$. The point is that $p \rightarrow 2$ if the maximal angle of $\Omega_{h}$ tends to $\pi$. This is exactly the case for $h \rightarrow 0$; therefore, we cannot deduce the boundedness of $\left\{\left\|\bar{\varphi}_{h}\right\|_{W^{2, p}\left(\Omega_{h}\right)}\right\}_{h>0}$ for any $p>2$.

By using the Stampacchia approach [11] we can derive a bound for $\left\|\bar{y}_{h}\right\|_{L^{\infty}\left(\Omega_{h}\right)}$ which is dependent on $\alpha, \beta$, and $a(\cdot, 0)$ but independent of $h$. Then, from (8.3) the boundedness of $\left\{\left\|\bar{\varphi}_{h}\right\|_{H^{2}\left(\Omega_{h}\right)}\right\}_{h>0}$ can be obtained. Now, from (8.4) we deduce

$$
\bar{u}_{h}(x)=\operatorname{Proj}_{[\alpha, \beta]}\left(-\frac{1}{N} \partial_{\nu_{h}} \bar{\varphi}_{h}(x)\right)=\max \left\{\alpha, \min \left\{-\frac{1}{N} \partial_{\nu_{h}} \bar{\varphi}_{h}(x), \beta\right\}\right\},
$$

which implies that $\bar{u}_{h} \in H^{1 / 2}\left(\Gamma_{h}\right)$ and the family $\left\{\left\|\bar{u}_{h}\right\|_{H^{1 / 2}\left(\Gamma_{h}\right)}\right\}_{h>0}$ is bounded. Finally, (8.2) leads to the boundedness of $\left\{\left\|\bar{y}_{h}\right\|_{H^{1}\left(\Omega_{h}\right)}\right\}_{h>0}$ as well.

Now, we prove the convergence of the local or global solutions of $\left(\mathrm{DP}_{h}\right)$ to the solutions of (DP) with $h \rightarrow 0$. To prove the convergence, first we establish the convergence of the solutions of the state and adjoint state equations.

Copyright $@$ by SIAM. Unauthorized reproduction of this article is prohibited. 
Theorem 8.3. Let $u \in H^{1 / 2}(\Gamma) \cap L^{\infty}(\Gamma)$ and $u_{h} \in L^{\infty}\left(\Gamma_{h}\right)$, with

$$
\max \left\{\|u\|_{L^{\infty}(\Gamma)},\left\|u_{h}\right\|_{L^{\infty}\left(\Gamma_{h}\right)}\right\} \leq M .
$$

Let $y_{u} \in H^{1}(\Omega) \cap L^{\infty}(\Omega)$ and $y_{h, u_{h}} \in H^{1 / 2}\left(\Omega_{h}\right) \cap L^{\infty}\left(\Omega_{h}\right)$ be the corresponding solutions of (7.1) and (8.1), respectively. Then there exists a constant $C_{M}>0$ independent of $h$ such that

$$
\left\|y_{u}-y_{h, u_{h}}\right\|_{H^{1 / 2}\left(\Omega_{h}\right)} \leq C_{M}\left(\left\|u-u_{h} \circ g_{h}^{-1}\right\|_{L^{2}(\Gamma)}+h\left[1+\|u\|_{H^{1 / 2}(\Gamma)}\right]\right) .
$$

Proof. Let us take $y_{h} \in H^{1 / 2}\left(\Omega_{h}\right) \cap L^{\infty}\left(\Omega_{h}\right)$ satisfying

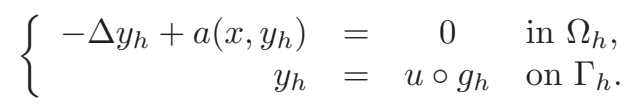

From (7.4) and (4.2) we get

$$
\begin{aligned}
\left\|y_{u}-y_{h, u_{h}}\right\|_{H^{1 / 2}\left(\Omega_{h}\right)} & \leq\left\|y_{u}-y_{h}\right\|_{H^{1 / 2}\left(\Omega_{h}\right)}+\left\|y_{h}-y_{h, u_{h}}\right\|_{H^{1 / 2}\left(\Omega_{h}\right)} \\
& \leq\left\|y_{u}-y_{h}\right\|_{H^{1 / 2}\left(\Omega_{h}\right)}+C\left\|u \circ g_{h}-u_{h}\right\|_{L^{2}\left(\Gamma_{h}\right)} \\
& \leq\left\|y_{u}-y_{h}\right\|_{H^{1 / 2}\left(\Omega_{h}\right)}+C\left\|u-u_{h} \circ g_{h}^{-1}\right\|_{L^{2}(\Gamma)} .
\end{aligned}
$$

Let us estimate $\phi_{h}=y_{u}-y_{h}$. By substraction of the equations satisfied by $y_{u}$ and $y_{h}$ and using the mean value theorem, we get

$$
\left\{\begin{array}{rlcl}
-\Delta \phi_{h}+\frac{\partial a}{\partial y}\left(x, w_{h}\right) \phi_{h} & = & 0 & \text { in } \Omega_{h}, \\
\phi_{h} & =y-u \circ g_{h} & & \text { on } \Gamma_{h},
\end{array}\right.
$$

where $w_{h}=y_{h}+\theta_{h}\left(y_{h, u_{h}}-y_{h}\right)$ and $0<\theta_{h}<1$. Now we have

$$
\left\|\phi_{h}\right\|_{H^{1 / 2}\left(\Omega_{h}\right)} \leq C\left\|y-u \circ g_{h}\right\|_{L^{2}\left(\Gamma_{h}\right)}=C\left\|y-y \circ g_{h}\right\|_{L^{2}\left(\Gamma_{h}\right)} .
$$

Finally, by using (5.9) we conclude

$$
\left\|\phi_{h}\right\|_{H^{1 / 2}\left(\Omega_{h}\right)} \leq C h\|y\|_{H^{1}(\Omega)} \leq C h\left(1+\|u\|_{H^{1 / 2}(\Gamma)}\right) .
$$

This inequality along with (8.9) proves (8.7).

Now we proceed with the analysis of the adjoint state equation. Let $\varphi_{u} \in H^{2}(\Omega)$ and $\varphi_{h, u_{h}} \in H^{2}\left(\Omega_{h}\right)$ be given as the solutions of the equations

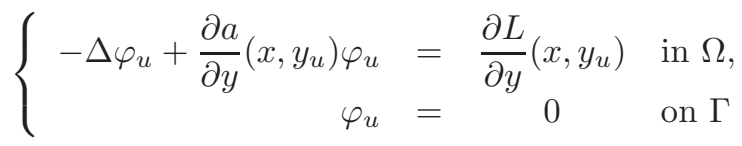

and

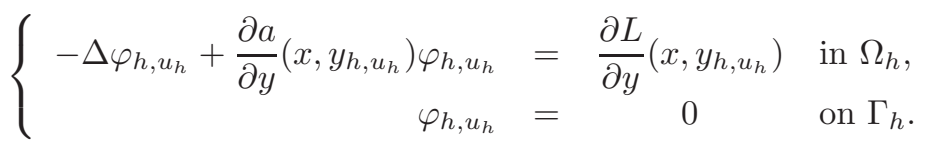

Then we have the following estimate.

TheOREM 8.4. Let $\left(u, y_{u}\right)$ and $\left(u_{h}, y_{h, u_{h}}\right)$ be as in Theorem 8.3. Let $\varphi_{u} \in H^{2}(\Omega)$ and $\varphi_{h, u_{h}} \in H^{2}\left(\Omega_{h}\right)$ be the corresponding solutions of (8.11) and (8.12), respectively.

Copyright (c) by SIAM. Unauthorized reproduction of this article is prohibited. 
Then there exists a constant $C_{M}>0$, independent of $h$, such that the following estimate holds:

$$
\left\|\varphi_{u}-\varphi_{h, u_{h}}\right\|_{H^{3 / 2}\left(\Omega_{h}\right)} \leq C_{M}\left(\left\|u-u_{h} \circ g_{h}^{-1}\right\|_{L^{2}(\Gamma)}+h\left[1+\|u\|_{H^{1 / 2}(\Gamma)}\right]\right) .
$$

Proof. Let us define $\phi_{h}=\varphi_{u}-\varphi_{h, u_{h}} \in H^{2}\left(\Omega_{h}\right)$. From (8.11) and (8.12) we get

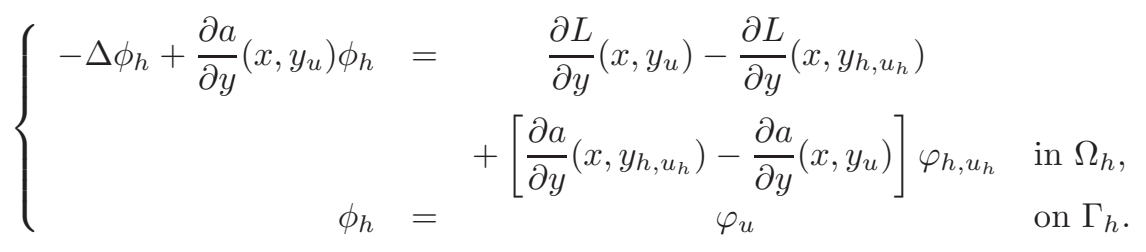

From assumption (D2), taking into account that $y_{u}$ and $y_{h, u_{h}}$ are bounded and using (8.7), we get (see Kenig [10])

$$
\begin{array}{r}
\left\|\phi_{h}\right\|_{H^{3 / 2}}\left(\Omega_{h}\right) \leq C\left(\left\|y_{u}-y_{h, u_{h}}\right\|_{L^{2}\left(\Omega_{h}\right)}+\left\|\varphi_{u}\right\|_{H^{1}\left(\Gamma_{h}\right)}\right) \\
\leq C(M)\left(\left\|u-u_{h} \circ g_{h}^{-1}\right\|_{L^{2}(\Gamma)}+h\left[1+\|u\|_{H^{1 / 2}(\Gamma)}\right]+\left\|\varphi_{u}\right\|_{H^{1}\left(\Gamma_{h}\right)}\right) .
\end{array}
$$

Let us estimate $\varphi_{u}$ in $H^{1}\left(\Gamma_{h}\right)$. The norm in $H^{1}\left(\Gamma_{h}\right)$ is given by

$$
\left\|\varphi_{u}\right\|_{H^{1}\left(\Gamma_{h}\right)}=\left\{\left\|\varphi_{u}\right\|_{L^{2}\left(\Gamma_{h}\right)}^{2}+\left\|\partial_{\tau_{h}} \varphi_{u}\right\|_{L^{2}\left(\Gamma_{h}\right)}^{2}\right\}^{1 / 2},
$$

where $\partial_{\tau_{h}} \varphi_{u}(x)=\nabla \varphi_{u}(x) \cdot \tau_{h}(x), \tau_{h}(x)$ being the unit tangent vector to $\Gamma_{h}$ at the point $x$; see section 4 . The estimate of the first term of the norm follows easily from (5.9) and the fact that $\varphi_{u} \circ g_{h}=0$ on $\Gamma_{h}$ :

$$
\left\|\varphi_{u}\right\|_{L^{2}\left(\Gamma_{h}\right)}=\left\|\varphi_{u}-\varphi_{u} \circ g_{h}\right\|_{L^{2}\left(\Gamma_{h}\right)} \leq C h^{2}\left\|\varphi_{u}\right\|_{H^{2}(\Omega)} \leq C(M) h^{2} .
$$

Now the $L^{2}(\Gamma)$ norm of the tangential derivative is estimated. To this end we observe that $\varphi_{u}=0$ on $\Gamma$; therefore, $\partial_{\tau} \varphi_{u}=0$ on $\Gamma$ as well. Thus, we also have $\left(\nabla \varphi_{u} \circ g_{h}\right) \cdot\left(\tau \circ g_{h}\right)=0$ on $\Gamma_{h}$. Hence

$$
\partial_{\tau_{h}} \varphi_{u}(x)=\left[\nabla \varphi_{u}(x)-\nabla \varphi_{u}\left(g_{h}(x)\right)\right] \tau_{h}(x)+\nabla \varphi_{u}\left(g_{h}(x)\right)\left[\tau_{h}(x)-\tau\left(g_{h}(x)\right)\right] .
$$

This along with (5.9) and (4.1) leads to

$$
\begin{aligned}
& \quad\left\|\partial_{\tau_{h}} \varphi_{u}\right\|_{L^{2}\left(\Gamma_{h}\right)} \leq\left\|\nabla \varphi_{u}-\nabla \varphi_{u} \circ g_{h}\right\|_{L^{2}\left(\Gamma_{h}\right)} \\
& +\left\|\varphi_{u}\right\|_{H^{2}(\Omega)}\left\|\tau_{h}-\tau \circ g_{h}\right\|_{L^{2}\left(\Gamma_{h}\right)} \leq C(M) h .
\end{aligned}
$$

Finally, (8.13) follows from (8.15), (8.16), and (8.17).

COROLLARY 8.5. Under the assumptions of Theorem 8.4, the following inequality holds:

$$
\left\|\partial_{\nu_{h}} \varphi_{u}-\partial_{\nu_{h}} \varphi_{h, u_{h}}\right\|_{L^{2}\left(\Gamma_{h}\right)} \leq C_{M}\left(\left\|u-u_{h} \circ g_{h}^{-1}\right\|_{L^{2}(\Gamma)}+h\left[1+\|u\|_{H^{1 / 2}(\Gamma)}\right]\right)
$$

for some $C_{M}>0$ independent of $h$.

Proof. It is enough to note that $\varphi_{u}-\varphi_{h, u_{h}} \in H^{3 / 2}\left(\Omega_{h}\right)$ and $\Delta\left(\varphi_{u}-\varphi_{h, u_{h}}\right) \in$ $L^{2}\left(\Omega_{h}\right)$; then $\partial_{\nu_{h}}\left(\varphi_{u}-\varphi_{h, u_{h}}\right) \in L^{2}\left(\Gamma_{h}\right)$, and we have

$$
\left\|\partial_{\nu_{h}}\left(\varphi_{u}-\varphi_{h, u_{h}}\right)\right\|_{L^{2}\left(\Gamma_{h}\right)} \leq\left\|\varphi_{u}-\varphi_{h, u_{h}}\right\|_{H^{3 / 2}\left(\Omega_{h}\right)}+\left\|\Delta\left(\varphi_{u}-\varphi_{h, u_{h}}\right)\right\|_{L^{2}\left(\Omega_{h}\right)} ;
$$

Copyright (c) by SIAM. Unauthorized reproduction of this article is prohibited. 
see [9] and [10]. From this inequality, assumption (D2), and estimates (8.7), (8.13), (8.14), and (8.15) we get

$$
\begin{array}{r}
\left\|\partial_{\nu_{h}}\left(\varphi_{u}-\varphi_{h, u_{h}}\right)\right\|_{L^{2}\left(\Gamma_{h}\right)} \leq C(M)\left(\left\|u-u_{h} \circ g_{h}^{-1}\right\|_{L^{2}(\Gamma)}+h\left[1+\|u\|_{H^{1 / 2}(\Gamma)}\right]\right) \\
+\left\|\frac{\partial a}{\partial y}\left(x, w_{h}\right)\right\|_{L^{2}\left(\Omega_{h}\right)}\left\|\varphi_{u}-\varphi_{h, u_{h}}\right\|_{L^{2}\left(\Omega_{h}\right)}+\left\|\frac{\partial L}{\partial y}\left(x, y_{u}\right)-\frac{\partial L}{\partial y}\left(x, y_{h, u_{h}}\right)\right\|_{L^{2}\left(\Omega_{h}\right)} \\
+\left\|\frac{\partial a}{\partial y}\left(x, y_{u}\right)-\frac{\partial a}{\partial y}\left(x, y_{h, u_{h}}\right)\right\|_{L^{2}\left(\Omega_{h}\right)}\left\|\varphi_{h, u_{h}}\right\|_{L^{2}\left(\Omega_{h}\right)} \\
\leq C_{M} h\left(\left\|u-u_{h} \circ g_{h}^{-1}\right\|_{L^{2}(\Gamma)}+h\left[1+\|u\|_{H^{1 / 2}(\Gamma)}\right]\right) .
\end{array}
$$

We complete this section by proving that the family of problems $\left(\mathrm{DP}_{h}\right)$ realizes a correct approximation of (DP). More precisely, we prove that the solutions of problems $\left(\mathrm{DP}_{h}\right)$ converge to the solutions of (DP). Reciprocally, we also prove that any strict local solution of (DP) can be approximated by a sequence of local solutions of problems $\left(\mathrm{DP}_{h}\right)$.

THEOREM 8.6. Let $\bar{u}_{h}$ be a solution of problem $\left(\mathrm{DP}_{h}\right)$. Then $\left\{\bar{u}_{h} \circ g_{h}^{-1}\right\}_{h>0}$ is a bounded family in $H^{1 / 2}(\Gamma)$. If $\bar{u}$ is a weak limit for a subsequence, still denoted in the same way, $\bar{u}_{h} \circ g_{h}^{-1} \rightarrow \bar{u}$ weakly in $H^{1 / 2}(\Gamma)$ with $h \rightarrow 0$, then $\bar{u}$ is a solution of problem (DP). Moreover,

$$
\lim _{h \rightarrow 0}\left\|\bar{y}-\bar{y}_{h}\right\|_{H^{1 / 2}\left(\Omega_{h}\right)}=0 \quad \text { and } \quad \lim _{h \rightarrow 0} J_{h}\left(\bar{u}_{h}\right) \rightarrow J(\bar{u}),
$$

where $\bar{y}$ and $\bar{y}_{h}$ denote the solutions of (7.1) and (8.1) corresponding to $\bar{u}$ and $\bar{u}_{h}$, respectively.

Proof. First, we recall the definition of the norm

$$
\begin{array}{r}
\left\|\bar{u}_{h} \circ g_{h}^{-1}\right\|_{H^{1 / 2}(\Gamma)}=\left\{\int_{\Gamma}\left|\bar{u}_{h}\left(g_{h}^{-1}(x)\right)\right|^{2} d \sigma(x)\right. \\
\left.+\int_{\Gamma} \int_{\Gamma} \frac{\left|\bar{u}_{h}\left(g_{h}^{-1}(x)\right)-\bar{u}_{h}\left(g_{h}^{-1}\left(x^{\prime}\right)\right)\right|^{2}}{\left|x-x^{\prime}\right|^{2}} d \sigma(x) d \sigma\left(x^{\prime}\right)\right\}^{1 / 2} .
\end{array}
$$

Let us estimate each of the two integrals. In Remark 8.2, we established the boundedness of $\left\{\left\|\bar{u}_{h}\right\|_{H^{1 / 2}\left(\Omega_{h}\right)}\right\}_{h>0}$. If we prove that

$$
\left\|\bar{u}_{h} \circ g_{h}^{-1}\right\|_{H^{1 / 2}(\Gamma)} \leq C\left\|\bar{u}_{h}\right\|_{H^{1 / 2}\left(\Gamma_{h}\right)}
$$

then we obtain that $\left\{\bar{u}_{h} \circ g_{h}^{-1}\right\}_{h>0}$ is bounded in $H^{1 / 2}(\Gamma)$. On the other hand, from (4.4) it follows that

$$
\begin{array}{r}
\left\|\bar{u}_{h} \circ g_{h}^{-1}\right\|_{L^{2}(\Gamma)}^{2}=\int_{\Gamma}\left|\bar{u}_{h}\left(g_{h}^{-1}(x)\right)\right|^{2} d \sigma(x) \\
=\int_{\Gamma_{h}}\left|\bar{u}_{h}(x)\right|^{2}\left|D g_{h}(x) \cdot \tau_{h}(x)\right| d \sigma_{h}(x) \leq C\left\|\bar{u}_{h}\right\|_{L^{2}\left(\Gamma_{h}\right)}^{2} .
\end{array}
$$

By a change of variables in the second integral of (8.19), in view of (4.4), we get

$$
\begin{aligned}
& \int_{\Gamma} \int_{\Gamma} \frac{\left|\bar{u}_{h}\left(g_{h}^{-1}(x)\right)-\bar{u}_{h}\left(g_{h}^{-1}\left(x^{\prime}\right)\right)\right|^{2}}{\left|x-x^{\prime}\right|^{2}} d \sigma(x) d \sigma\left(x^{\prime}\right) \\
& \leq C^{2} \int_{\Gamma_{h}} \int_{\Gamma_{h}} \frac{\left|\bar{u}_{h}(x)-\bar{u}_{h}\left(x^{\prime}\right)\right|^{2}}{\left|g_{h}(x)-g_{h}\left(x^{\prime}\right)\right|^{2}} d \sigma_{h}(x) d \sigma_{h}\left(x^{\prime}\right) .
\end{aligned}
$$

Copyright (c) by SIAM. Unauthorized reproduction of this article is prohibited. 
Let us show that $\left|x-x^{\prime}\right| \leq\left|g_{h}(x)-g_{h}\left(x^{\prime}\right)\right|$ for every $x, x^{\prime} \in \Gamma_{h}$. First, we assume that $x, x^{\prime} \in\left[x_{j}, x_{j+1}\right]$ for some $1 \leq j \leq N(h)$. Then

$$
x=x_{j}+t \tau_{j}, \quad g_{h}(x)=x+\phi_{j}(t) \nu_{j}, \quad x^{\prime}=x_{j}+t^{\prime} \tau_{j}, \quad \text { and } \quad g_{h}\left(x^{\prime}\right)=x^{\prime}+\phi_{j}\left(t^{\prime}\right) \nu_{j} .
$$

Therefore,

$\left|g_{h}(x)-g_{h}\left(x^{\prime}\right)\right|^{2}=\left|t-t^{\prime}\right|^{2}+\left|\phi_{j}(t)-\phi_{j}\left(t^{\prime}\right)\right|^{2}=\left|x-x^{\prime}\right|^{2}+\left|\phi_{j}(t)-\phi_{j}\left(t^{\prime}\right)\right|^{2} \geq\left|x-x^{\prime}\right|^{2}$.

Now, we assume that $x \in\left[x_{j}, x_{j+1}\right]$ and $x^{\prime} \in\left[x_{i}, x_{i+1}\right]$, with $i \neq j$. Since $\Omega$ is convex, there exist two points $\{\hat{x}\}=\left[x_{j}, x_{j+1}\right] \cap\left[g_{h}(x), g_{h}\left(x^{\prime}\right)\right]$ and $\left\{\hat{x}^{\prime}\right\}=\left[x_{i}, x_{i+1}\right] \cap$ $\left[g_{h}(x), g_{h}\left(x^{\prime}\right)\right]$. Moreover, we have

$$
\left|g_{h}(x)-g_{h}\left(x^{\prime}\right)\right|=\left|g_{h}(x)-\hat{x}\right|+\left|\hat{x}-\hat{x}^{\prime}\right|+\left|\hat{x}^{\prime}-g_{h}\left(x^{\prime}\right)\right| .
$$

On the other hand,

$$
g_{h}(x)-\hat{x}=\left(g_{h}(x)-x\right)+(x-\hat{x})=\phi_{j}(t) \nu_{j}+(t-\hat{t}) \tau_{j},
$$

which implies

$$
\left|g_{h}(x)-\hat{x}\right|^{2}=\left|g_{h}(x)-x\right|^{2}+|x-\hat{x}|^{2} \Rightarrow\left|g_{h}(x)-\hat{x}\right| \geq|x-\hat{x}| .
$$

Analogously, we can prove that $\left|g_{h}\left(x^{\prime}\right)-\hat{x}^{\prime}\right| \geq\left|x^{\prime}-\hat{x}^{\prime}\right|$. Finally, using (8.22), we obtain

$$
\left|g_{h}(x)-g_{h}\left(x^{\prime}\right)\right| \geq|x-\hat{x}|+\left|\hat{x}-\hat{x}^{\prime}\right|+\left|x^{\prime}-\hat{x}^{\prime}\right| \geq\left|x-x^{\prime}\right| .
$$

Using this inequality in (8.21), we conclude that

$$
\begin{aligned}
& \int_{\Gamma} \int_{\Gamma} \frac{\left|\bar{u}_{h}\left(g_{h}^{-1}(x)\right)-\bar{u}_{h}\left(g_{h}^{-1}\left(x^{\prime}\right)\right)\right|^{2}}{\left|x-x^{\prime}\right|^{2}} d \sigma(x) d \sigma\left(x^{\prime}\right) \\
& \leq C^{2} \int_{\Gamma_{h}} \int_{\Gamma_{h}} \frac{\left|\bar{u}_{h}(x)-\bar{u}_{h}\left(x^{\prime}\right)\right|^{2}}{\left|x-x^{\prime}\right|^{2}} d \sigma_{h}(x) d \sigma_{h}\left(x^{\prime}\right) .
\end{aligned}
$$

From (8.19), (8.20), and (8.23) it follows that

$$
\left\|\bar{u}_{h} \circ g_{h}^{-1}\right\|_{H^{1 / 2}(\Gamma)} \leq C^{\prime}\left\|\bar{u}_{h}\right\|_{H^{1 / 2}\left(\Gamma_{h}\right)} \leq C^{\prime \prime} .
$$

Therefore, there exists a subsequence and an element $\bar{u} \in H^{1 / 2}(\Gamma)$ such that $\bar{u}_{h} \circ g_{h}^{-1} \rightarrow$ $\bar{u}$ weakly in $H^{1 / 2}(\Gamma)$ with $h \rightarrow 0$. Since the embedding $H^{1 / 2}(\Gamma) \subset L^{2}(\Gamma)$ is compact, we have $\bar{u}_{h} \circ g_{h}^{-1} \rightarrow \bar{u}$ strongly in $L^{2}(\Gamma)$. It is obvious that $\alpha \leq \bar{u} \leq \beta$. Now, if we denote by $\bar{y}_{h}$ the states associated to $\bar{u}_{h}$ and by $\bar{y}$ the state associated to $\bar{u}$, we deduce from (8.7) that

$$
\lim _{h \rightarrow 0}\left\|\bar{y}-\bar{y}_{h}\right\|_{H^{1 / 2}\left(\Omega_{h}\right)}=0 \quad \text { and } \quad \exists C_{\alpha \beta}>0 \text { such that }\left\|\bar{y}_{h}\right\|_{L^{\infty}\left(\Omega_{h}\right)} \leq C_{\alpha \beta} \forall h .
$$

Hence, it is easy to prove that $J_{h}\left(\bar{u}_{h}\right) \rightarrow J(\bar{u})$. It remains to prove that $\bar{u}$ is a solution of (DP). Let us take any feasible control $u$ for (DP); then $u \circ g_{h}$ is also feasible for $\left(\mathrm{DP}_{h}\right)$. Therefore, since $\bar{u}_{h}$ is a solution of $\left(\mathrm{DP}_{h}\right)$, we obtain

$$
J(u)=\lim _{h \rightarrow 0} J_{h}\left(u \circ g_{h}\right) \geq \lim _{h \rightarrow 0} J_{h}\left(\bar{u}_{h}\right)=J(\bar{u}),
$$

Copyright $@$ by SIAM. Unauthorized reproduction of this article is prohibited. 
which completes the proof.

THEOREM 8.7. Let $\bar{u}$ be a strict local minimum of (DP); then there exists a family $\left\{\bar{u}_{h}\right\}$ such that each control $\bar{u}_{h}$ is a local minimum of $\left(\mathrm{DP}_{h}\right)$ and $\bar{u}_{h} \circ g_{h}^{-1} \rightarrow \bar{u}$ converges weakly in $H^{1 / 2}(\Gamma)$.

Proof. Let $\varepsilon>0$ be such that $\bar{u}$ is the unique global solution of problem

$$
\left(\mathrm{DP}_{\varepsilon}\right)\left\{\begin{array}{l}
\min J(u)=\int_{\Omega} L\left(x, y_{u}(x)\right) d x+\frac{N}{2} \int_{\Gamma} u^{2}(x) d \sigma(x) \\
\text { subject to }\left(y_{u}, u\right) \in\left(L^{\infty}(\Omega) \cap H^{1 / 2}(\Omega)\right) \times L^{2}(\Gamma), \\
\alpha \leq u(x) \leq \beta \text { for a.e. } x \in \Gamma \text { and }\|u-\bar{u}\|_{L^{2}(\Gamma)} \leq \varepsilon .
\end{array}\right.
$$

Now, for every $h$ we consider the problems

$$
\left(\mathrm{DP}_{h \varepsilon}\right)\left\{\begin{array}{l}
\min J_{h}(u)=\int_{\Omega_{h}} L\left(x, y_{h, u}(x)\right) d x+\frac{N}{2} \int_{\Gamma_{h}} u^{2}(x) d \sigma_{h}(x) \\
\text { subject to }\left(y_{h, u}, u\right) \in\left(L^{\infty}\left(\Omega_{h}\right) \cap H^{1 / 2}\left(\Omega_{h}\right)\right) \times L^{2}\left(\Gamma_{h}\right), \\
\alpha \leq u(x) \leq \beta \quad \text { for a.e. } x \in \Gamma_{h} \text { and }\left\|u \circ g_{h}^{-1}-\bar{u}\right\|_{L^{2}(\Gamma)} \leq \varepsilon
\end{array}\right.
$$

It is obvious that $\bar{u} \circ g_{h}$ is a feasible control for each problem $\left(\mathrm{DP}_{h \varepsilon}\right)$; therefore, there exists at least one solution $u_{h \varepsilon}$ of $\left(\mathrm{DP}_{h \varepsilon}\right)$. Let us show that $u_{h \varepsilon} \circ g_{h}^{-1} \rightarrow \bar{u}$ weakly in $H^{1 / 2}(\Gamma)$ with $h \rightarrow 0$.

Since $\left\{u_{h \varepsilon} \circ g_{h}^{-1}\right\}_{h>0}$ is bounded in $L^{\infty}(\Gamma)$, we can extract a subsequence, still denoted by the same symbol, and an element $\tilde{u} \in L^{\infty}(\Gamma)$ such that $u_{h \varepsilon} \circ g_{h}^{-1} \rightarrow \tilde{u}$ ${ }^{*}$ weakly in $L^{\infty}(\Gamma)$ with $h \rightarrow 0$. Let us denote by $y_{h \varepsilon} \in H^{1 / 2}\left(\Omega_{h}\right) \cap L^{\infty}\left(\Omega_{h}\right)$ the state associated to $u_{h \varepsilon}$ and consider an extension of $y_{h \varepsilon}$ to $\Omega$, still denoted by $y_{h \varepsilon}$, such that

$$
\left\|y_{h \varepsilon}\right\|_{H^{1 / 2}(\Omega)} \leq C\left\|y_{h \varepsilon}\right\|_{H^{1 / 2}\left(\Omega_{h}\right)} \text { and }\left\|y_{h \varepsilon}\right\|_{L^{\infty}(\Omega)} \leq C\left\|y_{h \varepsilon}\right\|_{L^{\infty}\left(\Omega_{h}\right)} \forall h .
$$

The boundedness of $\left\{u_{h \varepsilon} \circ g_{h}^{-1}\right\}$ in $L^{\infty}(\Gamma)$ implies that of $\left\{y_{h \varepsilon}\right\}$ in $H^{1 / 2}(\Omega)$. Therefore, by taking a subsequence, we can assume that

$$
y_{h \varepsilon} \rightarrow \tilde{y} \text { in } H^{1 / 2}(\Omega) \text { and } u_{h \varepsilon} \circ g_{h}^{-1} \rightarrow \tilde{u} \text { in } L^{2}(\Gamma) .
$$

We are going to prove that $\tilde{y}$ is the state associated to $\tilde{u}$. According to the definition given in section 7 , we have to prove that the following identity holds:

$$
\int_{\Omega}-\tilde{y} \Delta w d x+\int_{\Omega} a(x, \tilde{y}) w d x=\int_{\Gamma} \tilde{u} \partial_{\nu} w d \sigma \forall w \in H^{2}(\Omega) \cap H_{0}^{1}(\Omega) .
$$

For a given $w \in H^{2}(\Omega) \cap H_{0}^{1}(\Omega)$ we take $w_{h} \in H^{2}\left(\Omega_{h}\right) \cap H_{0}^{1}\left(\Omega_{h}\right)$, a unique solution of the Dirichlet problem

$$
\left\{\begin{array}{rlll}
-\Delta w_{h} & = & -\Delta w & \text { in } \Omega_{h} \\
w_{h} & = & 0 & \text { on } \Gamma_{h}
\end{array}\right.
$$

As in the proof of Theorem 8.4, we have

$$
\left\|w-w_{h}\right\|_{H^{3 / 2}\left(\Omega_{h}\right)} \leq C\|w\|_{H^{1}\left(\Omega_{h}\right)} \leq C h .
$$

Copyright (c) by SIAM. Unauthorized reproduction of this article is prohibited. 
Hence

$$
\begin{aligned}
\left\|\partial_{\nu_{h}} w-\partial_{\nu_{h}} w_{h}\right\|_{L^{2}\left(\Gamma_{h}\right)} & \leq C\left\{\left\|\Delta\left(w-w_{h}\right)\right\|_{L^{2}\left(\Omega_{h}\right)}+\left\|w-w_{h}\right\|_{H^{3 / 2}\left(\Omega_{h}\right)}\right\} \\
& =C\left\|w-w_{h}\right\|_{H^{3 / 2}\left(\Omega_{h}\right)} \leq C h .
\end{aligned}
$$

Since $y_{h \varepsilon}$ is the state associated to $u_{h \varepsilon}$, we have

$$
\int_{\Omega_{h}}-y_{h \varepsilon} \Delta w_{h} d x+\int_{\Omega_{h}} a\left(x, y_{h \varepsilon}\right) w_{h} d x=\int_{\Gamma_{h}} u_{h \varepsilon} \partial_{\nu_{h}} w_{h} d \sigma_{h} .
$$

In view of (8.25), this identity can be rewritten as follows:

$$
\int_{\Omega_{h}}-\Delta w y_{h \varepsilon} d x+\int_{\Omega_{h}} a\left(x, y_{h \varepsilon}\right) w_{h} d x=\int_{\Gamma_{h}} u_{h \varepsilon} \partial_{\nu_{h}} w_{h} d \sigma_{h} .
$$

Now we want to pass to the limit with $h \rightarrow 0$ in (8.28). Using the compactness of the embedding $H^{1 / 2}(\Omega) \subset L^{2}(\Omega)$ it is easy to pass to the limit in the first two integrals, which are also the first two integrals of (8.24). Let us consider the right-hand side term of (8.28). Applying (8.27), we get

$$
\int_{\Gamma_{h}} u_{h \varepsilon} \partial_{\nu_{h}} w_{h} d \sigma_{h}=\int_{\Gamma_{h}} u_{h \varepsilon} \partial_{\nu_{h}} w d \sigma_{h}+O(h) .
$$

Now from Lemma 8.8 below we deduce

$$
\int_{\Gamma_{h}} u_{h \varepsilon} \partial_{\nu_{h}} w d \sigma_{h}=\int_{\Gamma}\left(u_{h \varepsilon} \circ g_{h}^{-1}\right) \partial_{\nu} w d \sigma+O(h) .
$$

Finally, combining (8.29) and (8.30), we get

$$
\lim _{h \rightarrow 0} \int_{\Gamma_{h}} u_{h \varepsilon}(x) \partial_{\nu_{h}} w_{h} d \sigma_{h}=\int_{\Gamma} \tilde{u}(x) \partial_{\nu} w(x) d \sigma .
$$

Thus, we show that (8.24) follows from (8.28) by the limit passage.

Now, using that $u_{h \varepsilon} \circ g_{h}^{-1} \rightarrow \tilde{u}$ weakly in $L^{2}(\Gamma), y_{h \varepsilon} \rightarrow \tilde{y}$ strongly in $L^{2}(\Omega)$, $\left\{y_{h \varepsilon}\right\}_{h>0}$ is bounded in $L^{\infty}(\Omega)$, and the facts that $u_{h \varepsilon}$ is a solution of $\left(\mathrm{DP}_{h \varepsilon}\right)$ and $\bar{u} \circ g_{h}^{-1}$ is feasible for problems $\left(\mathrm{DP}_{h \varepsilon}\right)$, we obtain

$$
J(\tilde{u}) \leq \liminf _{h \rightarrow 0} J_{h}\left(u_{h \varepsilon}\right) \leq \liminf _{h \rightarrow 0} J_{h}\left(\bar{u} \circ g_{h}^{-1}\right) \leq \limsup _{h \rightarrow 0} J_{h}\left(\bar{u} \circ g_{h}^{-1}\right)=J(\bar{u}) .
$$

Since $\bar{u}$ is the unique solution of $\left(\mathrm{DP}_{\varepsilon}\right)$, the above inequality leads to $\tilde{u}=\bar{u}$ and $J_{h}\left(u_{h \varepsilon}\right) \rightarrow J(\bar{u})$, which implies

$$
\lim _{h \rightarrow 0} \int_{\Gamma_{h}} u_{h \varepsilon}^{2}(x) d \sigma_{h}(x)=\int_{\Gamma} \bar{u}^{2}(x) d \sigma(x) .
$$

Using (4.3)

$$
\lim _{h \rightarrow 0} \int_{\Gamma}\left(u_{h \varepsilon} \circ g_{h}^{-1}\right)^{2}(x) d \sigma(x)=\int_{\Gamma} \bar{u}^{2}(x) d \sigma(x) .
$$

This identity and the weak convergence imply the strong convergence $u_{h \varepsilon} \circ g_{h}^{-1} \rightarrow \bar{u}$ in $L^{2}(\Gamma)$. The first consequence of this strong convergence is that the constraint $\| u \circ$ 
$g_{h}^{-1}-\bar{u} \|_{L^{2}(\Gamma)} \leq \varepsilon$ is not active at the controls $u_{h \varepsilon}$ for $h$ small enough. Therefore, $u_{h \varepsilon}$ is a local minimum of problem $\left(\mathrm{DP}_{h}\right)$ for every $h$ small enough. Since $\left\{\left\|u_{h \varepsilon}\right\|_{L^{2}\left(\Gamma_{h}\right)}\right\}$ is bounded, we can argue as in the proof of Theorem 8.6 and conclude that $\left\{u_{h \varepsilon} \circ g_{h}^{-1}\right\}$ is bounded in $H^{1 / 2}(\Gamma)$ and hence $u_{h \varepsilon} \circ g_{h}^{-1} \rightarrow \bar{u}$ weakly in $H^{1 / 2}(\Gamma)$ with $h \rightarrow 0$.

LEMma 8.8. Let $w \in H^{2}(\Omega)$ and $v \in L^{2}(\Gamma)$; then there exists a constant $C>0$ independent of $w$ and $v$ such that

$$
\left|\int_{\Gamma} \partial_{\nu} w v d \sigma-\int_{\Gamma_{h}} \partial_{\nu_{h}} w\left(v \circ g_{h}\right) d \sigma_{h}\right| \leq C h\|w\|_{H^{2}(\Omega)}\|v\|_{L^{2}(\Gamma)} .
$$

Proof. First, we observe that (4.3) implies that

$$
\begin{array}{r}
\left|\int_{\Gamma}[\nabla w(x) \cdot \nu(x)] v(x) d \sigma(x)-\int_{\Gamma_{h}}\left[\nabla w\left(g_{h}(x)\right) \cdot \nu\left(g_{h}(x)\right)\right] v\left(g_{h}(x)\right) d \sigma_{h}(x)\right| \\
\leq C_{\Gamma} h^{2} \int_{\Gamma}|\nabla w(x) \cdot \nu(x) \| v(x)| d \sigma(x) \\
\leq C_{\Gamma} h^{2}\left\|\partial_{\nu} w\right\|_{L^{2}(\Gamma)}\|v\|_{L^{2}(\Gamma)} \leq C h^{2}\|w\|_{H^{2}(\Omega)}\|v\|_{L^{2}(\Gamma)} .
\end{array}
$$

On the other hand,

$$
\begin{array}{r}
\int_{\Gamma_{h}}\left[\nabla w(x) \cdot \nu_{h}(x)\right] v\left(g_{h}(x)\right) d \sigma_{h}(x)-\int_{\Gamma_{h}}\left[\nabla w\left(g_{h}(x)\right) \cdot \nu\left(g_{h}(x)\right)\right] v\left(g_{h}(x)\right) d \sigma_{h}(x) \\
=\int_{\Gamma_{h}}\left[\nabla w(x) \cdot\left(\nu_{h}(x)-\nu\left(g_{h}(x)\right)\right)\right] v\left(g_{h}(x)\right) d \sigma_{h}(x) \\
+\int_{\Gamma_{h}}\left[\nabla w(x)-\nabla w\left(g_{h}(x)\right)\right] \cdot \nu\left(g_{h}(x)\right) v\left(g_{h}(x)\right) d \sigma_{h}(x) .
\end{array}
$$

From this identity we get, in view of (4.1), (4.2), and (5.9),

$$
\begin{array}{r}
\left|\int_{\Gamma_{h}}\left[\nabla w(x) \cdot \nu_{h}(x)\right] v\left(g_{h}(x)\right) d \sigma_{h}(x)-\int_{\Gamma_{h}}\left[\nabla w\left(g_{h}(x)\right) \cdot \nu\left(g_{h}(x)\right)\right] v\left(g_{h}(x)\right) d \sigma_{h}(x)\right| \\
\leq C h\|w\|_{H^{2}(\Omega)}\|v\|_{L^{2}(\Gamma)} .
\end{array}
$$

Now, (8.32) and (8.33) imply (8.31).

9. Error estimates for problems $\left(\mathbf{D P}_{\boldsymbol{h}}\right)$. In this section we assume that $\bar{u}_{h}$ is a local minimum of $\left(\mathrm{DP}_{h}\right)$ such that $\bar{u}_{h} \circ g_{h}^{-1}$ converges weakly in $H^{1 / 2}(\Gamma)$ to a local minimum $\bar{u}$ of (DP) with $h \rightarrow 0$; see Theorems 8.6 and 8.7. The goal of this section is to derive an estimate for $\left\|\bar{u}-\bar{u}_{h} \circ g_{h}^{-1}\right\|_{L^{2}(\Gamma)}$, which is established in the following theorem.

TheOrem 9.1. Let $\bar{u}$ and $\bar{u}_{h}$ be as above, and let us denote by $\bar{y}, \bar{y}_{h}$ and $\bar{\varphi}, \bar{\varphi}_{h}$ the states and adjoint states associated to $\bar{u}$ and $\bar{u}_{h}$, respectively. Let us assume that the second order sufficient optimality condition (7.13) is fulfilled for $\bar{u}$. Then there exists a constant $C$, independent of $h$, such that the following estimates hold:

$$
\left\|\bar{u}-\bar{u}_{h} \circ g_{h}^{-1}\right\|_{L^{2}(\Gamma)}+\left\|\bar{y}-\bar{y}_{h}\right\|_{H^{1 / 2}\left(\Omega_{h}\right)}+\left\|\bar{\varphi}-\bar{\varphi}_{h}\right\|_{H^{3 / 2}\left(\Omega_{h}\right)} \leq C h .
$$

Before proving this theorem we provide a preliminary result. The proof of Lemma 9.2 is inspired by [5, Lemma 7.2]; however, there are some important differences.

Copyright $@$ by SIAM. Unauthorized reproduction of this article is prohibited. 
Lemma 9.2. Let $\mu>0$ be taken from Theorem 7.4. Then there exists $h_{0}>0$ such that

$$
\frac{1}{2} \min \{N, \mu\}\left\|\bar{u}_{h} \circ g_{h}^{-1}-\bar{u}\right\|_{L^{2}(\Gamma)}^{2} \leq\left(J^{\prime}\left(\bar{u}_{h} \circ g_{h}^{-1}\right)-J^{\prime}(\bar{u})\right)\left(\bar{u}_{h} \circ g_{h}^{-1}-\bar{u}\right) .
$$

Proof. By applying the mean value theorem there is an intermediate element $\hat{u}_{h}=\bar{u}+\theta_{h}\left(\bar{u}_{h} \circ g_{h}^{-1}-\bar{u}\right)$ such that

$$
\left(J^{\prime}\left(\bar{u}_{h} \circ g_{h}^{-1}\right)-J^{\prime}(\bar{u})\right)\left(\bar{u}_{h} \circ g_{h}^{-1}-\bar{u}\right)=J^{\prime \prime}\left(\hat{u}_{h}\right)\left(\bar{u}_{h} \circ g_{h}^{-1}-\bar{u}\right)^{2} .
$$

Let us take

$$
v_{h}=\frac{1}{\left\|\bar{u}_{h} \circ g_{h}^{-1}-\bar{u}\right\|_{L^{2}(\Gamma)}}\left(\bar{u}_{h} \circ g_{h}^{-1}-\bar{u}\right) .
$$

Taking a subsequence, if necessary, we can assume that $v_{h} \rightarrow v$ weakly in $L^{2}(\Gamma)$. We show that $v$ belongs to the critical cone $C_{\bar{u}}$ defined in section 7 . First, observe that $v$ satisfies the sign condition (7.12) since every element $v_{h}$ satisfies the same condition. Let us prove that $v(x)=0$ if $N \bar{u}(x)-\partial_{\nu} \bar{\varphi}(x) \neq 0$. To this end it is enough to establish the limit passage

$$
\lim _{h \rightarrow 0} \int_{\Gamma_{h}}\left(N \bar{u}_{h}-\partial_{\nu_{h}} \bar{\varphi}_{h}\right)\left(v_{h} \circ g_{h}\right) d \sigma_{h}=\int_{\Gamma}\left(N \bar{u}-\partial_{\nu} \bar{\varphi}\right) v d \sigma .
$$

Indeed, from (9.4) we deduce, in view of (8.4), that

$$
\begin{array}{r}
\int_{\Gamma}\left|N \bar{u}-\partial_{\nu} \bar{\varphi}\right||v| d \sigma=\int_{\Gamma}\left(N \bar{u}-\partial_{\nu} \bar{\varphi}\right) v d \sigma \\
=\lim _{h \rightarrow 0} \frac{1}{\left\|\bar{u}_{h} \circ g_{h}^{-1}-\bar{u}\right\|_{L^{2}(\Gamma)}} \int_{\Gamma_{h}}\left(N \bar{u}_{h}-\partial_{\nu_{h}} \bar{\varphi}_{h}\right)\left(\bar{u}_{h}-\bar{u} \circ g_{h}\right) d \sigma_{h} \leq 0,
\end{array}
$$

which proves the required property. Let us show (9.4). By the strong convergence $\bar{u}_{h} \circ g_{h}^{-1} \rightarrow \bar{u}$ in $L^{2}(\Gamma)$ combined with (8.18) and (4.2), we have

$$
\begin{array}{r}
\left|\int_{\Gamma_{h}}\left(N \bar{u}_{h}-\partial_{\nu_{h}} \bar{\varphi}_{h}\right)\left(v_{h} \circ g_{h}\right) d \sigma_{h}-\int_{\Gamma_{h}}\left(N \bar{u}_{h}-\partial_{\nu_{h}} \bar{\varphi}\right)\left(v_{h} \circ g_{h}\right) d \sigma_{h}\right| \\
\leq\left\|\partial_{\nu_{h}} \bar{\varphi}_{h}-\partial_{\nu_{h}} \bar{\varphi}\right\|_{L^{2}\left(\Gamma_{h}\right)}\left\|v_{h} \circ g_{h}\right\|_{L^{2}\left(\Gamma_{h}\right)} \\
\leq C_{M}\left(\left\|\bar{u}_{h} \circ g_{h}^{-1}-\bar{u}\right\|_{L^{2}(\Gamma)}+h\left[1+\|u\|_{H^{1 / 2}(\Gamma)}\right]\right)\left\|v_{h}\right\|_{L^{2}(\Gamma)} \\
=C_{M}\left(\left\|\bar{u}_{h} \circ g_{h}^{-1}-\bar{u}\right\|_{L^{2}(\Gamma)}+h\left[1+\|u\|_{\left.H^{1 / 2}(\Gamma)\right]}\right]\right) \rightarrow 0 \text { with } h \rightarrow 0 .
\end{array}
$$

On the other hand, from Lemma 8.8 we get

$$
\int_{\Gamma_{h}} \partial_{\nu_{h}} \bar{\varphi}\left(v_{h} \circ g_{h}\right) d \sigma_{h}=\int_{\Gamma} \partial_{\nu} \bar{\varphi} v_{h} d \sigma+O(h) \rightarrow \int_{\Gamma} \partial_{\nu} \bar{\varphi} v d \sigma \text { with } h \rightarrow 0 .
$$

Finally, from (4.3) we obtain

$$
\int_{\Gamma_{h}} \bar{u}_{h}\left(v_{h} \circ g_{h}\right) d \sigma_{h}=\int_{\Gamma}\left(\bar{u}_{h} \circ g_{h}^{-1}\right) v_{h} d \sigma+O(h) \rightarrow \int_{\Gamma} \bar{u} v d \sigma \quad \text { with } h \rightarrow 0 .
$$

Thus, (9.4) follows from (9.5), (9.6), and (9.7).

Copyright $@$ by SIAM. Unauthorized reproduction of this article is prohibited. 
Now by the definition of $v_{h}$ and (7.7), (7.26), we get

$$
\begin{array}{r}
\lim _{h \rightarrow 0} J^{\prime \prime}\left(\hat{u}_{h}\right) v_{h}^{2}=\lim _{h \rightarrow 0}\left\{\int_{\Omega}\left[\frac{\partial^{2} L}{\partial y^{2}}\left(x, y_{\hat{u}_{h}}\right)-\varphi_{\hat{u}_{h}} \frac{\partial^{2} a}{\partial y^{2}}\left(x, y_{\hat{u}_{h}}\right)\right] z_{v_{h}}^{2} d x+N\right\} \\
=\int_{\Omega}\left[\frac{\partial^{2} L}{\partial y^{2}}(x, \bar{y})-\bar{\varphi} \frac{\partial^{2} a}{\partial y^{2}}(x, \bar{y})\right] z_{v}^{2} d x+N \\
=J^{\prime \prime}(\bar{u}) v^{2}+N\left(1-\|v\|_{L^{2}(\Gamma)}^{2}\right) \geq N+(\mu-N)\|v\|_{L^{2}(\Gamma)}^{2} .
\end{array}
$$

Taking into account that $\|v\|_{L^{2}(\Gamma)} \leq 1$, the above inequality leads to

$$
\lim _{h \rightarrow 0} J^{\prime \prime}\left(\hat{u}_{h}\right) v_{h}^{2} \geq \min \{\mu, N\}>0,
$$

which proves the existence of $h_{0}>0$ such that

$$
J^{\prime \prime}\left(\hat{u}_{h}\right) v_{h}^{2} \geq \frac{1}{2} \min \{\mu, N\} \quad \forall h<h_{0} .
$$

From this inequality, by the definition of $v_{h}$ and (9.3), we deduce (9.2), which completes the proof.

Proof of Theorem 9.1. By taking $v=\bar{u}_{h} \circ g_{h}^{-1}$ in (7.11) and $v_{h}=\bar{u} \circ g_{h}$ in (8.4), we get

$$
J^{\prime}(\bar{u})\left(\bar{u}_{h} \circ g_{h}^{-1}-\bar{u}\right)=\int_{\Gamma}\left(N \bar{u}-\partial_{\nu} \bar{\varphi}\right)\left(\bar{u}_{h} \circ g_{h}^{-1}-\bar{u}\right) d \sigma \geq 0
$$

and

$$
J_{h}^{\prime}\left(\bar{u}_{h}\right)\left(\bar{u} \circ g_{h}-\bar{u}_{h}\right)=\int_{\Gamma_{h}}\left(N \bar{u}_{h}-\partial_{\nu_{h}} \bar{\varphi}_{h}\right)\left(\bar{u} \circ g_{h}-\bar{u}_{h}\right) d \sigma_{h} \geq 0 .
$$

We rewrite inequality (9.9) as follows:

$$
J^{\prime}\left(\bar{u}_{h} \circ g_{h}^{-1}\right)\left(\bar{u}-\bar{u}_{h} \circ g_{h}^{-1}\right)+\left[J_{h}^{\prime}\left(\bar{u}_{h}\right)\left(\bar{u} \circ g_{h}-\bar{u}_{h}\right)-J^{\prime}\left(\bar{u}_{h} \circ g_{h}^{-1}\right)\left(\bar{u}-\bar{u}_{h} \circ g_{h}^{-1}\right)\right] \geq 0 .
$$

From (9.8) and (9.10) we obtain

$$
\left[J^{\prime}\left(\bar{u}_{h} \circ g_{n}^{-1}\right)-J^{\prime}(\bar{u})\right]\left(\bar{u}_{h} \circ g_{h}^{-1}-\bar{u}\right) \leq J_{h}^{\prime}\left(\bar{u}_{h}\right)\left(\bar{u} \circ g_{h}-\bar{u}_{h}\right)-J^{\prime}\left(\bar{u}_{h} \circ g_{h}^{-1}\right)\left(\bar{u}-\bar{u}_{h} \circ g_{h}^{-1}\right) .
$$

Now, from (9.2) we deduce

$$
\frac{1}{2} \min \{N, \mu\}\left\|\bar{u}_{h} \circ g_{h}^{-1}-\bar{u}\right\|_{L^{2}(\Gamma)}^{2} \leq J_{h}^{\prime}\left(\bar{u}_{h}\right)\left(\bar{u} \circ g_{h}-\bar{u}_{h}\right)-J^{\prime}\left(\bar{u}_{h} \circ g_{h}^{-1}\right)\left(\bar{u}-\bar{u}_{h} \circ g_{h}^{-1}\right) .
$$

It remains to derive an estimate for the right-hand side of (9.11). To this end, we introduce $y \in H^{1}(\Omega) \cap L^{\infty}(\Omega)$ and $\varphi \in H^{2}(\Omega)$ as the solutions of the equations

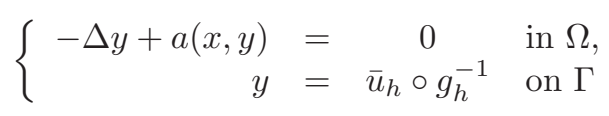

and

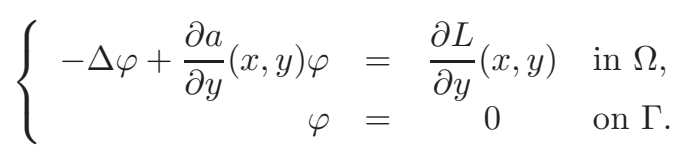

Copyright (c) by SIAM. Unauthorized reproduction of this article is prohibited. 
Then we have

$$
J^{\prime}\left(\bar{u}_{h} \circ g_{h}^{-1}\right)\left(\bar{u}-\bar{u}_{h} \circ g_{h}^{-1}\right)=\int_{\Gamma}\left(N \bar{u}_{h} \circ g_{h}^{-1}-\partial_{\nu} \varphi\right)\left(\bar{u}-\bar{u}_{h} \circ g_{h}^{-1}\right) d \sigma .
$$

From (9.9) we get

$$
=\left|\int_{\Gamma_{h}}\left(N \bar{u}_{h}-\partial_{\nu_{h}} \bar{\varphi}_{h}\right)\left(\bar{u} \circ g_{h}-\bar{u}_{h}\right) d \sigma_{h}-\int_{\Gamma}\left(N \bar{u}_{h} \circ g_{h}^{-1}-\partial_{\nu} \varphi\right)\left(\bar{u}-\bar{u}_{h} \circ g_{h}^{-1}\right) d \sigma\right| .
$$

Using (4.3) we obtain

$$
\begin{array}{r}
\left|\int_{\Gamma_{h}} \bar{u}_{h}\left(\bar{u} \circ g_{h}-\bar{u}_{h}\right) d \sigma_{h}-\int_{\Gamma} \bar{u}_{h} \circ g_{h}^{-1}\left(\bar{u}-\bar{u}_{h} \circ g_{h}^{-1}\right) d \sigma\right| \\
\leq C h^{2} \int_{\Gamma}\left|\bar{u}_{h} \circ g_{h}^{-1}\right|\left|\bar{u}-\bar{u}_{h} \circ g_{h}^{-1}\right| d \sigma \\
\leq C h^{2}\left\|\bar{u}_{h} \circ g_{h}^{-1}\right\|_{L^{2}(\Gamma)}\left\|\bar{u}-\bar{u}_{h} \circ g_{h}^{-1}\right\|_{L^{2}(\Gamma)} \leq C h^{2}\left\|\bar{u}-\bar{u}_{h} \circ g_{h}^{-1}\right\|_{L^{2}(\Gamma)} .
\end{array}
$$

On the other hand, from (8.18), Lemma 8.8, and (4.3), we get

$$
\begin{array}{r}
\left|\int_{\Gamma_{h}} \partial_{\nu_{h}} \bar{\varphi}_{h}\left(\bar{u} \circ g_{h}-\bar{u}_{h}\right) d \sigma_{h}-\int_{\Gamma} \partial_{\nu} \varphi\left(\bar{u}-\bar{u}_{h} \circ g_{h}^{-1}\right) d \sigma\right| \\
\left.\leq\left\|\partial_{\nu_{h}} \bar{\varphi}_{h}-\partial_{\nu_{h}} \varphi\right\|_{L^{2}\left(\Gamma_{h}\right.}\right)\left\|\bar{u} \circ g_{h}^{-1}-\bar{u}_{h}\right\|_{L^{2}\left(\Gamma_{h}\right)} \\
+\left|\int_{\Gamma_{h}} \partial_{\nu_{h}} \varphi\left(\bar{u} \circ g_{h}-\bar{u}_{h}\right) d \sigma_{h}-\int_{\Gamma} \partial_{\nu} \varphi\left(\bar{u}-\bar{u}_{h} \circ g_{h}^{-1}\right) d \sigma\right| \\
\leq C h\left\|\bar{u}-\bar{u}_{h} \circ g_{h}^{-1}\right\|_{L^{2}(\Gamma)} .
\end{array}
$$

Thus, from (9.11), (9.15), (9.16), and (9.17), we conclude

$$
\left\|\bar{u}-\bar{u}_{h} \circ g_{h}^{-1}\right\|_{L^{2}(\Gamma)} \leq C h .
$$

The remaining estimates of (9.1) follow from the above estimate, (8.7), and (8.13).

\section{REFERENCES}

[1] J. Bramble And J. King, A robust finite element method for nonhomogeneous Dirichlet problems in domains with curved boundaries, Math. Comp., 63 (1994), pp. 1-17.

[2] E. Casas and M. Mateos, Second order optimality conditions for semilinear elliptic control problems with finitely many state constraints, SIAM J. Control Optim., 40 (2002), pp. 1431-1454.

[3] E. Casas And M. Mateos, Error estimates for the numerical approximation of Neumann control problems, Comput. Optim. Appl., 39 (2008), pp. 265-295.

[4] E. Casas, M. Mateos, and F. Tröltzsch, Error estimates for the numerical approximation of boundary semilinear elliptic control problems, Comput. Optim. Appl., 31 (2005), pp. 193219.

[5] E. CASAS AND J.-P. RAYMOND, Error estimates for the numerical approximation of Dirichlet boundary control for semilinear elliptic equations, SIAM J. Control Optim., 45 (2006), pp. 1586-1611.

[6] K. Deckelnick, A. Günther, And M. Hinze, Finite element approximation of Dirichlet boundary control for elliptic PDEs on two- and three-dimensional curved domains, SIAM J. Control Optim., 48 (2009), pp. 2798-2819.

[7] P. Grisvard, Elliptic Problems in Nonsmooth Domains, Pitman, Boston, London, Melbourne, 1985.

Copyright (c) by SIAM. Unauthorized reproduction of this article is prohibited. 
[8] M. Hinze, A variational discretization concept in control constrained optimization: The linearquadratic case, Comput. Optim. Appl., 30 (2005), pp. 45-61.

[9] D. Jerison and C. Kenig, The Neumann problem on Lipschitz domains, Bull. Amer. Math. Soc. (N.S.), 4 (1981), pp. 203-207.

[10] C. Kenig, Harmonic Analysis Techniques for Second Order Elliptic Boundary Value Problems, CBMS Regional Conference Series in Mathematics 83, AMS, Providence, RI, 1994.

[11] G. Stampacchia, Le problème de Dirichlet pour les équations elliptiques du second ordre à coefficients discontinus, Ann. Inst. Fourier (Grenoble), 15 (1965), pp. 189-258.

Copyright (c) by SIAM. Unauthorized reproduction of this article is prohibited. 